\title{
A level set-based approach for modeling cellular rearrangements in tissue morphogenesis
}

\author{
Rhudaina Mohammad \\ University of the Philippines https://orcid.org/0000-0002-9803-4350 \\ Hideki Murakawa \\ Ryukoku University \\ Karel Svadlenka ( $\sim$ karel@math.kyoto-u.ac.jp ) \\ Kyoto University \\ Hideru Togashi \\ Kobe University Graduate School of Medicine https://orcid.org/0000-0002-3967-5940
}

Article

Keywords:

Posted Date: October 7th, 2021

DOl: https://doi.org/10.21203/rs.3.rs-43983/v4

License: (9) This work is licensed under a Creative Commons Attribution 4.0 International License.

Read Full License

Version of Record: A version of this preprint was published at Communications Biology on March 18th, 2022. See the published version at https://doi.org/10.1038/s42003-022-03174-6. 


\title{
A level set-based approach for modeling cellular rearrangements in tissue morphogenesis
}

\author{
Rhudaina Z. Mohammad ${ }^{\mathrm{a}, \mathrm{f}}$, Hideki Murakawa ${ }^{\mathrm{b}}$, Karel Švadlenka ${ }^{\mathrm{a}, \mathrm{c}, 1}$, Hideru Togashi $^{\mathrm{d}, \mathrm{e}}$ \\ ${ }^{a}$ Department of Mathematics, Graduate School of Science, Kyoto University, Kyoto, Japan \\ ${ }^{b}$ Applied Mathematics and Informatics Course, Faculty of Advanced Science and Technology, Ryukoku University, Otsu, \\ Japan \\ ${ }^{c}$ Mathematical Institute, Czech Academy of Sciences, Prague, Czech Republic \\ ${ }^{d}$ Department of Biochemistry and Molecular Biology, Kobe University Graduate School of Medicine, Kobe, Japan \\ e JST PRESTO (Precursory Research for Embryonic Science and Technology), Kobe, Japan \\ ${ }^{f}$ Institute of Mathematics, College of Science, University of the Philippines Diliman, Quezon City, Philippines
}

\begin{abstract}
Among morphological phenomena, cellular patterns in developing sensory epithelia have gained attention in recent years. Although physical models for cellular rearrangements are well-established thanks to a large bulk of experimental work, their computational implementation lacks solid mathematical background and involves experimentally unreachable parameters. Here we introduce a level set-based computational framework as a tool to rigorously investigate evolving cellular patterns, and study its mathematical and computational properties. We illustrate that a significant feature of the method is its ability to correctly handle complex topology changes, including frequent cell intercalations. Combining this accurate numerical scheme with an established mathematical model, we show that the new framework features minimum possible number of parameters and is capable of reproducing a wide range of tissue morphological phenomena, such as cell sorting, engulfment or internalization. In particular, thanks to precise mathematical treatment of cellular intercalations, this method is the first to successfully simulate experimentally observed development of cellular mosaic patterns in sensory epithelia.
\end{abstract}

\section{Introduction}

Understanding the mechanisms of tissue morphogenesis - how interacting processes generate the shape and structure of an organism - is at the forefront of researches in developmental biology. Mathematical modeling

Email addresses: rmohammad@math.upd.edu.ph (Rhudaina Z. Mohammad), murakawa@math.ryukoku.ac.jp (Hideki

Murakawa), karel@math.kyoto-u.ac.jp (Karel Švadlenka), htogashi@med.kobe-u.ac.jp (Hideru Togashi)

${ }^{1}$ Corresponding Author 
is a powerful tool to investigate how local cell-cell interactions affect tissue-level morphology. In general, a successful model requires two main ingredients: a mathematical model represented by a set of governing equations based on physical principles, and a computational method to solve them. Building each of these two components is a different process - a simple physical model may not necessarily be easy to compute. In this paper, we focus on the computational aspect of modeling the morphology of epithelial tissues, where the gap between a model and its numerical realization is prominent. Indeed, the physical model usually measures adhesion or tension forces along cellular interfaces and thus is straightforward, while its computation presents challenging tasks due to topology changes in cellular rearrangement - a highly nontrivial problem from the viewpoint of mathematics.

Formation of cellular patterns in tissues often involves frequent neighbor swaps (topology changes). Cell intercalation, a typical process of this type, requires a distinctive combination of mechanisms, including adhesive changes that allow cells to rearrange, or cytoskeletal events through which cells exert the forces needed for cell neighbor exchange. Brodland (2002) in his Differential Interfacial Tension Hypothesis (DITH) proposed that relative intensities of interfacial tensions, which are defined as a combination of cell-cell adhesion, contraction of cell membranes and other forces, lead to self-driven rearrangement of embryonic cells. To analyze the effect of such factors in tissue self-organization, a number of mathematical models have been proposed with the assumption that tissue evolves via a succession of quasi-equilibrium states, that is, cell shapes are described by their instantaneous state of lowest energy (Steinberg, 1963). In this article, we follow this line and focus on a class of models which neglect inertial effects and treat the evolution of a cellular aggregate from the viewpoint of free energy minimization principle; see Methods and Supplementary Section B for more details.

Existing computational approaches realizing the above-mentioned free energy minimization include, among others, vertex dynamics model, cellular Potts model, front-tracking and finite element methods (see Supplementary Section A for a brief overview of their differences). In this paper, we present another class of numerical methods based on the implicit level set representation of the shape of cell-cell junctions. Here an evolving junction is a contour of a time-dependent function on a fixed spatial grid. Recent mathematical results on the evolution of level sets of interfacial networks via energy gradient descent (Esedoḡlu and Otto, 2015) allow for a precise computation of this evolution including topology changes. We draw attention to this computational method for its advantages and to show that in certain problems it may perform better than well-established schemes. Namely, we claim that besides its ability to accurately handle topology changes, the level set approach eliminates nonphysical parameters.

Although each specific morphogenetic phenomenon involves a large number of biological and physical factors, it is a well-accepted understanding in the modeling community that it is not reasonable to construct models having a large number of factors as model parameters. The reason is simply that correlation analysis becomes 
prohibitively complicated with increasing number of parameters, especially in living systems: if a model has a sufficiently large number of parameters, their suitable tuning can produce essentially arbitrary results and the analysis becomes pointless. This leads researchers to pin down biologically significant components in a given phenomenon, and build a model with only those components as parameters. However, the aforementioned models incorporate a number of parameters which cannot be omitted and have only vague physical interpretations. For example, to deal with topological changes due to cellular intercalations one performs junctional rearrangements in the vertex dynamics model through simple operations, such as T1, T2, T3 transitions (Fletcher et al. 2013); and in the finite element-based method through a boundary "flip" algorithm (Brodland, 2004), which all require additional parameters.

A typical example of a phenomenon where the above concerns become relevant is formation of cellular patterns in morphogenesis of sensory epithelia. These epithelia show regular mosaic or checkerboard patterns that crystallize through continuous intercalations of sensory and supporting cells. In the work of Katsunuma et al. (2016), it was hypothesized that varying adhesion strengths between participating cells is the decisive factor determining the mosaic pattern.

However, it was found that frequent occurrence of cell intercalations precludes the application of vertex dynamics method to support the hypothesis through simulations. Our proposed level set scheme addresses all the main causes of failure of vertex dynamics in this case, namely, inaccurate handling of topology changes, presence of nonphysical parameters, and inability of expressing complex curved shapes of cell junctions with largely different cell sizes, leading to successful reproduction of experimentally observed cellular patterns. Our results give a strong indication of the correctness of the hypothesis that differential interfacial tension plays the main role in formation of epithelial tissues.

\section{Results}

\subsection{Mathematical Model and Level Set-Based Approach}

We present an outline of the mathematical model and numerical algorithm (see Methods for a systematic explanation). In choosing a model, we adhere to the context of DITH (Brodland, 2002). Cells in an aggregate are expressed as closed regions $\mathcal{C}_{1}, \ldots, \mathcal{C}_{N}$, and cell-cell junctions are defined by $\gamma_{i j}:=\mathcal{C}_{i} \cap \mathcal{C}_{j}$. In cases where there is an extracellular space, we denote this medium by the same symbol $\mathcal{C}_{k}$ and its cell-medium interface by $\gamma_{i k}$. Surface tension between $i$ th and $j$ th cell, that is equivalent to interfacial free energy density, is denoted by $\sigma_{i j}$ (see Supplementary Section B for a discussion on its physical meaning). We consider cellular rearrangement as the $L^{2}$-gradient flow of the free energy

$$
E\left(\mathcal{C}_{1}, \ldots, \mathcal{C}_{N}\right)=\sum_{i \neq j} \sigma_{i j} \operatorname{Area}\left(\gamma_{i j}\right)
$$


constrained by each cell's prescribed volume $V_{\ell}^{0}(\ell=1,2, \ldots, N)$. We assume that each cell exactly preserves its volume and that apoptosis does not occur but this simplification can be made more realistic by allowing varying volumes of cells, see Supplementary Section B. Thus, our mathematical model is standard and conceptually simple but its numerical implementation is not obvious.

We propose a level set-based scheme for numerical realization of this model, based on ideas in Esedoḡlu and Otto (2015). The core of Esedoḡlu-Otto's algorithm is to solve heat equations and to extract level sets of their solutions, which can be easily and efficiently implemented. Moreover, the convergence of this algorithm is supported in Laux and Otto (2016) from mathematical point of view even for the problem containing multiple junctions. In order to apply Esedoğlu-Otto algorithm to simulations of evolving cell aggregates, two issues need to be addressed: volumes of cells have to be preserved or controlled, and cells have to be prevented from splitting. We have implemented volume control by combining the Esedoḡlu-Otto algorithm with auction dynamics (Jacobs et al., 2018), which in essence finds suitable contour heights by simulating an auction (see Figure 1 and the intuitive explanation of the auction algorithm in Methods). The second issue relates to the absence of any device in the original algorithm that would keep cells as connected sets. It may then happen that a cell splits into two parts and one part suddenly appears in a distant part of the aggregate. To avoid such behavior, we implement a localization on the bidding process in auction dynamics. Figure 1 depicts the main steps of the resulting algorithm.

In Methods, we provide further details of the algorithm, summarize its known mathematical properties such as stability and convergence, and give a detailed account of parameters used. The important conclusion is that outputs of the proposed algorithm depend only on the physical parameters $\sigma_{i j}$, apart from the unavoidable space-time discretization steps $\delta x, \delta t$. Since theoretical results on convergence and stability for the full volume-controlled problem including localization are not available, we also present a computational analysis of the proposed method showing that it has the desired properties, in particular convergence even across topology changes. Moreover, we perform a series of numerical tests to contrast the properties of our level set-based algorithm with those of the vertex dynamics algorithm, a commonly-used method for cell intercalations in developmental biology, showing that the level set-based method yields better results at the price of higher computational cost. We defer the details on the setup and results of these experiments to Supplementary Section D, Supplementary Section E and Supplementary Section F.

In Supplementary Section G, we report that our level set-based method generates expected results for standard morphogenetic benchmarks, namely cell sorting, mixing and formation of checkerboard or football patterns. Finally, in Supplementary Section H. performing a simulation of an encapsulation phenomenon in embryo morphogenesis, we show that our method can handle simulations in higher dimensions without major technical or theoretical complications. 


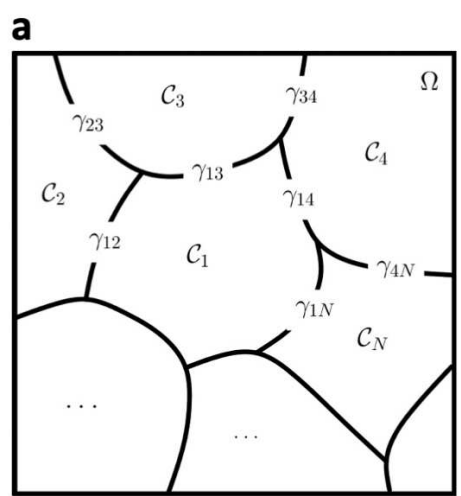

\section{b}

b1 Initial condition

Cell regions are expressed by their characteristic functions.

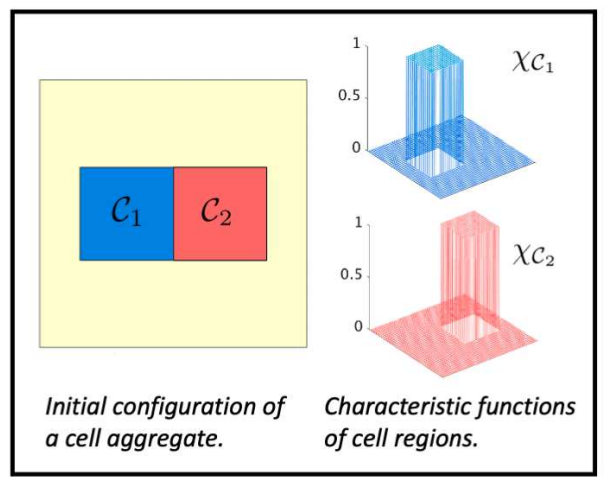

\section{b2 Convolution}

Heat equations are solved and preferences $\psi_{i}$ are calculated.

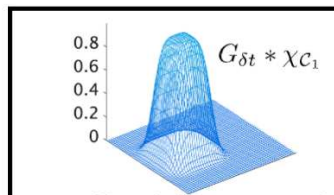

Diffused characteristic functions,

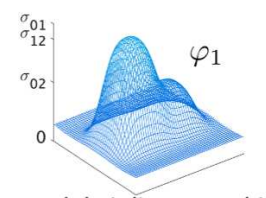

and their linear combinations yielding $\psi_{i}=1-\varphi_{i}$.

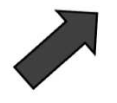

b3 Auction dynamics

Contours of new cell regions are obtained based on $\psi_{i}$.
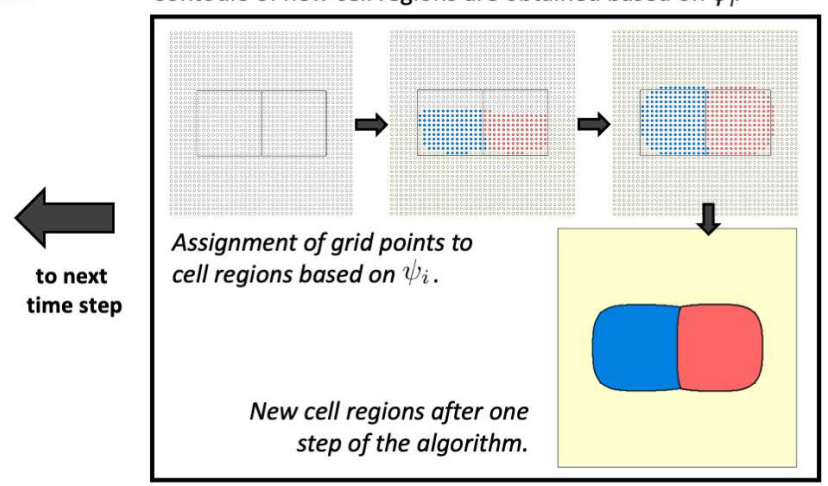

Figure 1: Basic notation and outline of the algorithm. a, A cellular aggregate is represented as a bounded domain $\Omega$ partitioned into $N$ cells $\mathcal{C}_{1}, \ldots, \mathcal{C}_{N}$. The boundary between cells $\mathcal{C}_{i}$ and $\mathcal{C}_{j}$ denotes the cell-cell junction $\gamma_{i j}$. b. Three main steps of the algorithm generating an approximation of the cellular rearrangement at the next time step $\delta t$ : (b1 Initial condition) Given an initial cell aggregate, it is represented by characteristic functions $\chi_{c_{i}}, i=1, \ldots, N$, of respective cell regions on a discrete grid (functions corresponding to the yellow region are not shown). (b2 Convolution) Characteristic functions are diffused via solving heat equation, that is, convolutions with Gaussian kernel $G_{\delta t} * \chi_{\mathcal{C}_{i}}$ are computed for every $i$, followed by linear combination of diffused functions with weights corresponding to interfacial tensions $\sigma_{i j}$, i.e., $\varphi_{i}=\sum_{j=1}^{N} \sigma_{i j}\left(G_{\delta t} * \chi_{\mathcal{C}_{j}}\right)$. The values $\psi_{i}(x)=1-\varphi_{i}(x)$ then determine the preference of each (grid) node $x$ for belonging to cell region $\mathcal{C}_{i}$. (b3 Localized auction dynamics) Grid points are assigned to cell regions in several sweeps of auction-like process where grid points bid to neighboring cells based on the preferences $\psi_{i}$. Level sets of the final bid $b(x)$ determine the cell configuration at the next time step $\delta t$, which optimizes preferences while preserving cell volumes (see also Movie S5.

\subsection{Simulation of Tissue Engulfment}

With the purpose of further validating the new method from the biological viewpoint, we present an application to a morphogenetic phenomenon in a medium, where one cell mass, say red, totally engulfs another cell mass, say blue. Following the viewpoint of DITH, Brodland and Chen (2000) proposed that 
for the blue cell mass to be enveloped by the red cell mass, a sufficient condition is $\sigma_{\mathrm{BR}}<\sigma_{\mathrm{BG}}-\sigma_{\mathrm{RG}}$ (here $\sigma_{\mathrm{BR}}$ means the interfacial tension between blue and red cells; analogously for $\sigma_{\mathrm{BG}}, \sigma_{\mathrm{RG}}$, where $\mathrm{G}$ means the gray medium). Moreover, for such engulfment to continue, it is necessary to have $\sigma_{\mathrm{BR}}<\sigma_{\mathrm{BG}}$ (Brodland, 2002). With this in mind, consider an initial configuration of 10 blue cells, 40 red cells, and a gray medium on a computational domain $\Omega=[0,1] \times[0,1]$ discretized uniformly into $M=500 \times 500$ points with periodic boundary conditions. We take interfacial tensions $\sigma_{\mathrm{BB}}=\sigma_{\mathrm{RR}}=1.0, \sigma_{\mathrm{BG}}=7.5, \sigma_{\mathrm{RG}}=3.5, \sigma_{\mathrm{BR}}=7.5$, then linearly change $\sigma_{\mathrm{BR}}$ from 7.5 to 2.5 over 100 time steps, keeping $\sigma_{\mathrm{BR}}=2.5$ for the remaining time of the simulation, cf., Brodland (2002). We then generate the corresponding aggregate evolution using our level set-based scheme with time step $\delta t=0.001$. Observe that the final configuration results in total engulfment of the blue cell mass by the red cell mass (Figure 2 and Movie S1]. This is not easily achieved by finite element-based simulations, cf., Figure 8 of Brodland (2002), which yield unnaturally distorted shapes of cells, particularly, in the region where the blue cell mass is engulfed by red cells. The artifacts in the FEM-based approach emerge from explicit treatment of cellular intercalations using an ad hoc boundary "flip" algorithm. This demonstrates the superiority of our method over the finite element-based scheme in handling such topology changes.
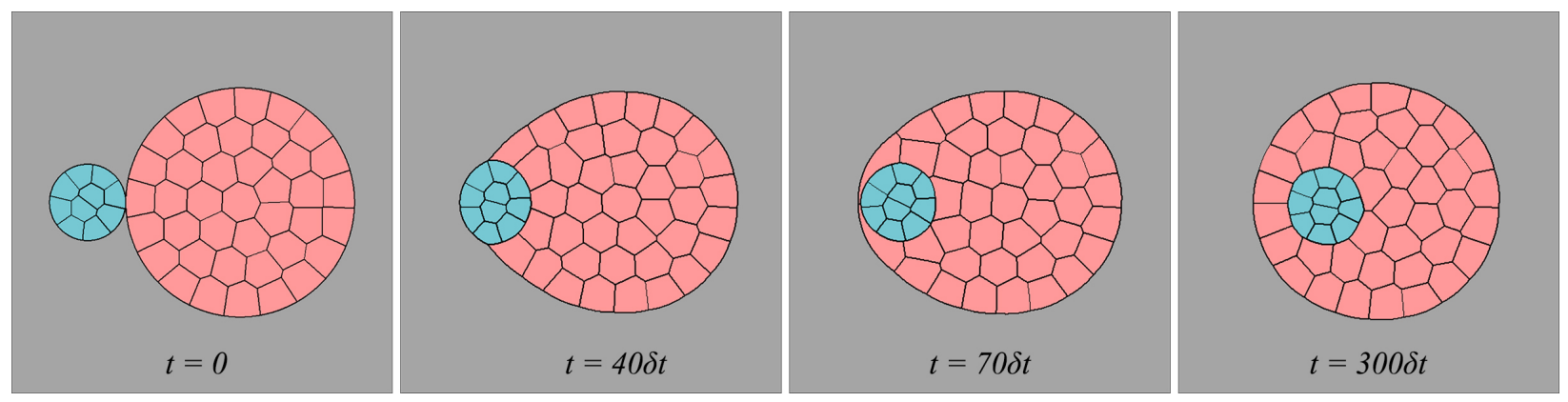

Figure 2: Total engulfment via level set-based model. An initial aggregate of 10 blue and 40 red cells surrounded by a gray medium and its evolution generated by our level set-based method, resulting in total engulfment of the blue cell mass by the red cell mass. Here the interfacial tensions are $\sigma_{\mathrm{BB}}=\sigma_{\mathrm{RR}}=1.0, \sigma_{\mathrm{BG}}=7.5, \sigma_{\mathrm{RG}}=3.5$, and $\sigma_{\mathrm{BR}}$ linearly changing from 7.5 at time $t=0$ to 2.5 at time $t=100 \delta t$ and then kept constant.

\subsection{Simulation of Cellular Rearrangement in Olfactory Epithelia}

In the following two sections, we introduce novel simulation results showing the potential of the level set method in computationally reproducing observed cellular patterns in developing sensory systems, in particular, olfactory and auditory epithelia. Such morphologies involve curved cell junctions, significantly different sizes of participating cells and frequent topology changes. The level set approach is successful in dealing with this complexity not only because it allows for a wide range of geometrical patterns but also because it implicitly satisfies the precise contact angles at tricellular junctions, which is indispensable for theoretically correct realization of cellular intercalation processes. 
Vertebrates possess highly developed sense organs, responsible for detecting information about different environments and converting extracellular stimuli into electrical signals which are mediated by specialized sensory epithelia (Togashi, 2016). Mosaic cellular patterns have been observed in various sensory epithelia, such as football pattern in the olfactory epithelium and checkerboard pattern in the auditory epithelium. These regular mosaic patterns are evolutionary conserved among a wide range of species and thought to be important in sensory functions.

The olfactory epithelium, which is located inside the nasal cavity, is a specialized sensory epithelium that is involved in odor perception. When the luminal surface of the olfactory epithelium is observed from the apical side, ciliated olfactory cells (OCs) and several types of supporting cells (SCs) are arranged in a unique mosaic pattern (Figure 3 $\mathrm{p}$ ). In a developing olfactory epithelium, this pattern formation is accompanied by cellular rearrangements from embryonic day 14 (E14) to postnatal day 1 (P1) (Figure 33), which is thought to be driven by the different adhesion between OCs and SCs (Katsunuma et al. 2016). To estimate cell-cell adhesion strength, we use the distributional patterns of $\beta$-catenin intensities since in this case, cadherin-dependent affinity is the major component of cell-cell adhesion. During the rearrangement, adhesion strengths between OCs and SCs (OS), SCs and SCs (SS), and OCs and OCs (OO) continuously change (Figure 3b).

Using distributional patterns of experimentally measured $\beta$-catenin intensities, cf. Katsunuma et al. (2016), we apply our level set-based approach to simulate cellular rearrangements in the olfactory epithelium from E14 to P1 stage (Figure 35). We consider an initial aggregate of 26 OCs (red) and 24 SCs (blue) on a square domain $\Omega=[0,1] \times[0,1]$ with periodic boundary conditions. As observed in biological experiments, OCs are approximately 10 times smaller in size than SCs and tend to cluster around tricellular SC-SC junctions at E14 (see Figure 33). We discretize the domain uniformly into $M=500 \times 500$ points, take time step $\delta t=0.0008$, and set interfacial tension $\sigma_{i j}$ as the reciprocal of cell-cell adhesion strength $\alpha_{i j}$, measured in terms of $\beta$-catenin intensity.

Figure 35 and Movie S2 show the simulation of cellular rearrangement of a developing olfactory epithelium. Comparing this to biological experiments, we see that the level set-based model was able to capture overall cellular rearrangements in the embryonic stage. In particular, at E14 stage, OCs cluster at the tricellular SCSC junctions; then from E16 to E18, OCs separate and move along SC-SC junctions. The only noticeable difference is that OCs at tricellular junctions are not as round as in the experimental results. Lastly, postnatal simulation indicates that $\beta$-catenin and, thus, cadherin-dependent cell-cell adhesion, is not the only contributing factor for cellular rearrangement in olfactory epithelium. Indeed, the discrepancy observed in postnatal stages is caused by an insufficiency in the model parameters rather than by the level setbased numerical method or the model itself, since if the parameters of the model are chosen in a suitable hypothetical way, cellular patterns matching those observed experimentally are obtained by the level set 


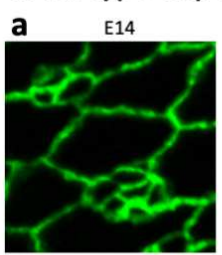

C $\quad t=0$
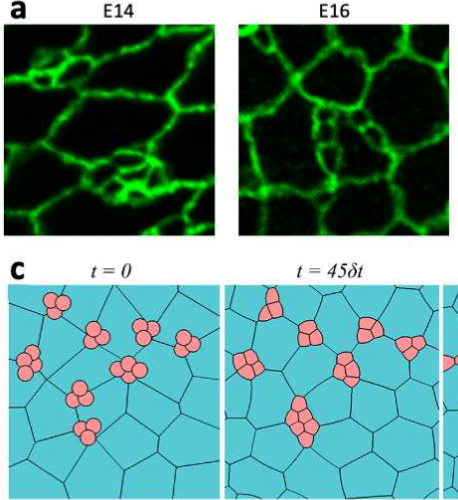

$t=45 \delta t$
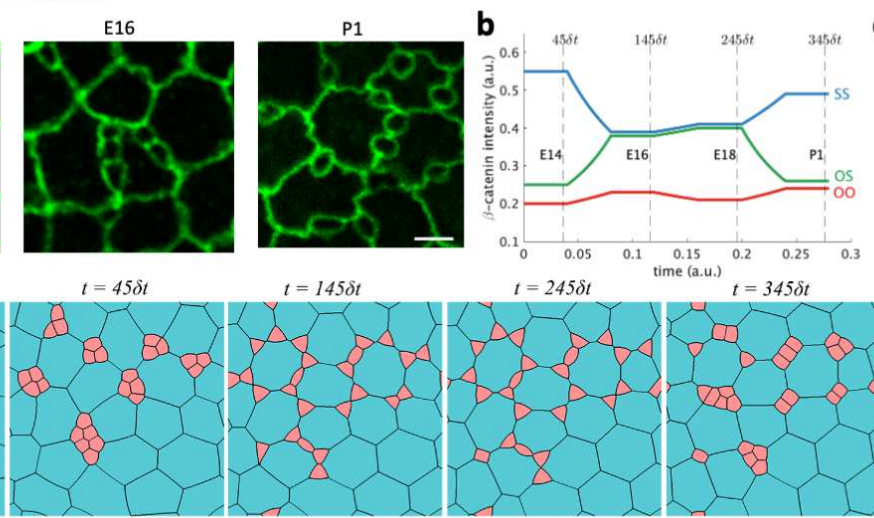

$t=245 \delta t$
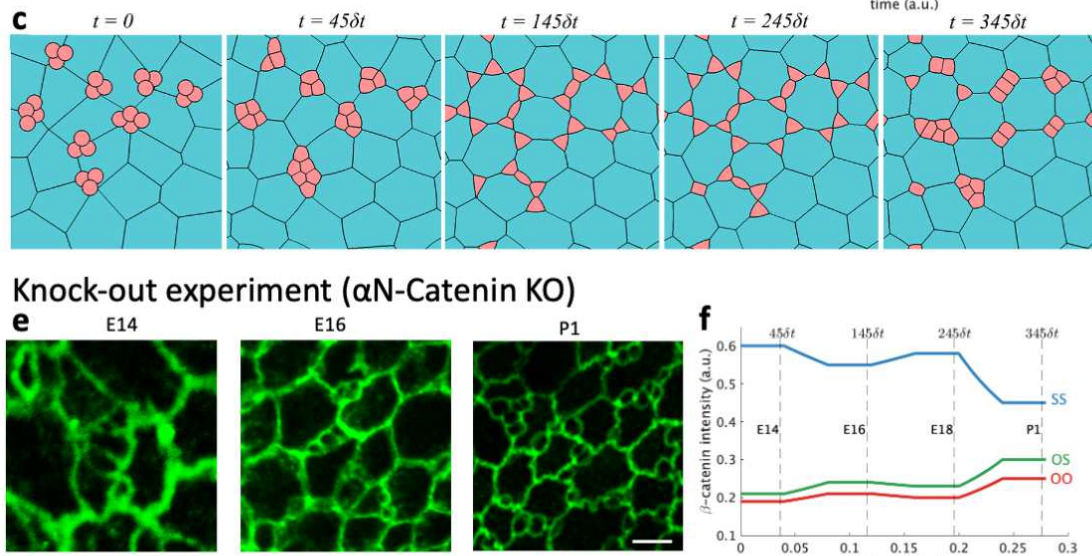

g $t=0$

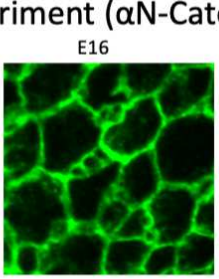

$t=45 \delta t$
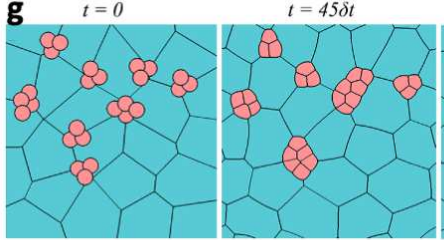

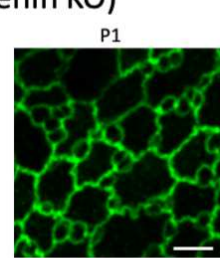

$t=145 \delta t$

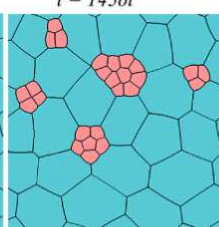

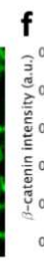

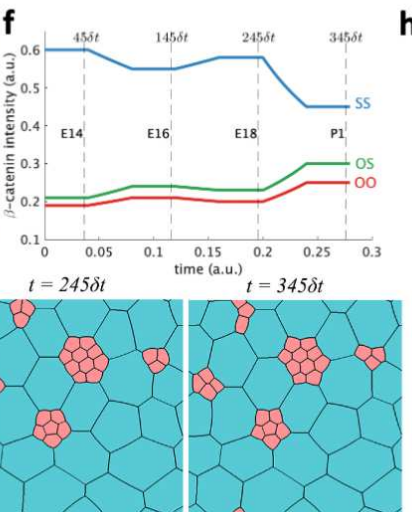

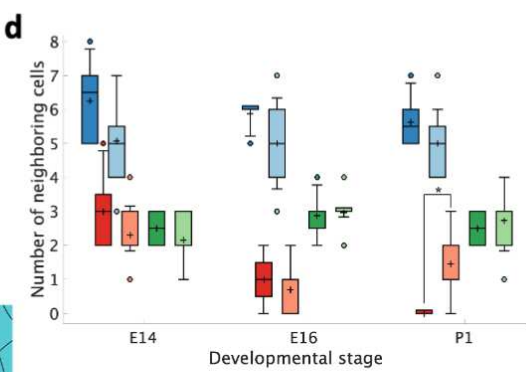

$\begin{array}{lll}\text { SC-SC experiment } & \text { OC-OC experiment } & \text { OC-SC experiment } \\ \text { SC-SC simulation } & \text { OC-OC simulation } & \text { OC-SC simulation }\end{array}$

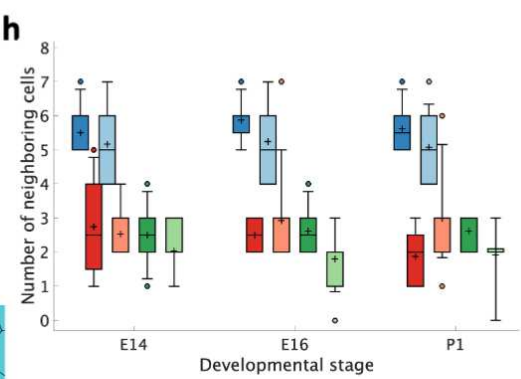

Figure 3: Actual images and simulation results of a developing olfactory epithelium. a, Immunostaining for junctional marker (ZO-1) on the apical surface of the mouse OE from embryonic days (E14, E16) to postnatal day (P1) (C)2016 Katsunuma et al., originally published in Journal of Cell Biology). Scale bar $5 \mu \mathrm{m}$ (all figures have the same scale). $\mathbf{b}$, Plot of relative intensity of $\beta$-catenin accumulations at OO, OS, and SS junctions during development, obtained by interpolation of $\beta$-catenin intensity mean values at time instants E14, E16, E18 and P1 reported in Figure S2 of Katsunuma et al. (2016). Moreover, the initial (E14) and final (P1) values were kept constant until the cellular pattern equilibrated. c, A simulation of cellular rearrangement of an olfactory epithelium from initial aggregate of 26 red OCs and 24 blue SCs to embryonic stages E14, E16, E18 until postnatal P1 stage obtained by the level set-based model. d, Cell neighbor counts obtained from three representative actual images and one simulation image for $t=45 \delta t, 145 \delta t$ and $345 \delta t$ shown in $\mathbf{c}$. The boxes in boxplots show first and third quartiles, whiskers show 9th and 91 th percentiles and + sign shows the mean. Significant difference $(p<0.01$, marked by asterisk) was found in OC-OC neighbors at P1 stage ( $n=8$ (experiment); $n=26$ (simulation)). e-h, Corresponding results for $\alpha$ N-catenin $\mathrm{KO}$ mouse model based on experimentally measured $\beta$-catenin intensities.

approach. To generate cellular patterns similar to those observed in olfactory epithelium at P1 stage, we took the same initial configuration and the same discretization parameters as above. The interfacial tensions $\sigma_{\mathrm{SS}}, \sigma_{\mathrm{OS}}$ and $\sigma_{\mathrm{OO}}$ were evolved as shown in Figure 4 ; $;$ namely, until stage E18 interfacial tensions identical to those obtained from measurements as presented in Figure $3 \mathrm{~b}$ are adopted, while at further stages $\sigma_{\mathrm{OS}}$ and 
$\sigma_{\mathrm{OO}}$ were significantly increased so that eventually $\sigma_{\mathrm{OS}}$ is twice as larger than $\sigma_{\mathrm{SS}}$ and $\sigma_{\mathrm{OO}}$ is twice as larger than $\sigma_{\mathrm{OS}}$. This intends to imitate the hypothesized prominent activity of cytoskeleton within olfactory cells. The resulting evolution of the aggregate is shown in Figure $4 \mathrm{~b}$ and Movie S3, exhibiting a clear agreement with the actual pattern depicted in Figure 3 a (P1), which is further quantified in Figure 4c by analyzing the numbers of neighboring cell types. In particular, olfactory cells are separated one from another and are located either at tricellular junctions or in the middle of boundary between supporting cells.
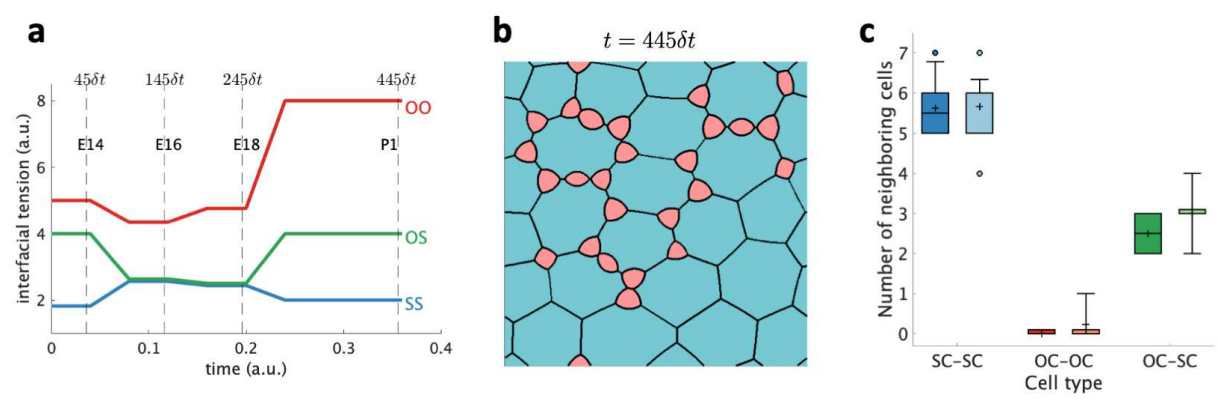

Figure 4: Simulation of developing olfactory epithelium until postnatal stage. a, Evolution of interfacial tensions which is based on measurements up to embryonic stage E18 but is selected hypothetically for postnatal stage

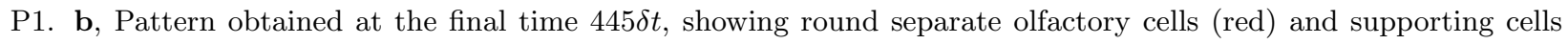
(blue) arranged in a pattern similar to experimental image in Figure 3 a (P1). Patterns obtained at earlier times until $245 \delta t$ are identical to those in Figure 3. c, Boxplot of neighboring cell counts corresponding to this hypothetical simulation. Counts for the simulation image $\mathbf{b}$ are shown on the right in light colors. The meaning of the boxes and markers is the same as in Figure 3 d.

To assess how well our numerical simulation captures experimentally observed results, we also consider cellular patterns observed in developing olfactory epithelium derived from $\alpha \mathrm{N}$-catenin knock-out mice. Here adhesiveness at OC-SC junctions is significantly decreased, which severely affects the resulting cellular pattern (Figure 3 ). Using distributional patterns of $\beta$-catenin intensities measured from $\alpha \mathrm{N}$-catenin $\mathrm{KO}$ mice, we applied our level set-based approach to simulate cellular rearrangements in the olfactory epithelium from E14 to P1 stage (see Figure 3s and Movie S2). We then analyze both wild-type (WT) and knock-out (KO) cellular patterns by counting neighboring OCs and SCs at randomly selected cells in three actual images, and at each computational cell of the simulations shown in Figure 35,g. To be precise, for each olfactory cell, number of neighboring supporting cells (OC-SC) and olfactory cells (OC-OC), and for each supporting cell, number of neighboring supporting cells (SC-SC) is counted. We confirmed that the counts do not significantly depend on the initial condition by running 4 more simulations, starting from randomly generated initial configurations of 26 OCs and 24 SCs. Figure $3 \mathrm{~d}$,h reveals that the mean cell morphology for both WT and KO obtained in silico matches well with experimental counterparts in the embryonic stage, but a significant discrepancy is observed in the postnatal stage. We conclude that the level-set based numerical method is able to simulate complex cell behaviors in evolving olfactory epithelium across all stages until $\mathrm{P} 1$, while the physical model based on $\beta$-catenin mediated adhesion captures well the 
phenomenon only in the embryonic stages.

\subsection{Simulation of Checkerboard Pattern Formation in Auditory Epithelia}

Another example of unique cellular pattern formation in tissues is the auditory epithelium, which is responsible for hearing. It is composed of mechanosensory hair cells (HCs), equipped with stereocilia that sense sound, and supporting cells (SCs) that help the functions of hair cells. These cells rearrange to form a checkerboard pattern from embryonic day 14 (E14) to 16 (E16) (Figure 5a,b). At E18, HCs are arranged in ordered rows, and each $\mathrm{HC}$ is separated from one another by a SC, forming an alternating mosaic pattern. In the auditory epithelium, nectin-1 and nectin-3 are complementarily expressed in HCs and SCs, respectively. During the above-mentioned developmental stages, molecular interactions occur between nectin-1 on HCs and nectin-3 on SCs, where the heterophilic molecular interaction between nectin-1 and -3 is much stronger than the homophilic interactions of nectins between the same type of cells. This biased cell-cell adhesion is responsible for the checkerboard assembly of cells. On the other hand, absence of nectin-3 (Nectin-3 KO) eliminated this bias in cell-cell adhesion, leading to cell rearrangement including attachments between HCs, and an overall disruption of the checkerboard pattern (Togashi et al., 2011. Togashi, 2016).

To unravel the mechanism of this cell rearrangement, we applied the level set-based method to an initial configuration of $12 \mathrm{HCs}$ (red), 12 SCs (blue), and one pillar cell (gray) aligned, as observed in experiments, at the top and bottom of a rectangular domain $\Omega=[0,1] \times\left[0, \frac{5}{8}\right]$ with periodic boundary conditions both on left and right boundaries, and on top and bottom boundaries (Figure 55). We discretized $\Omega$ uniformly into $M=480 \times 300$ points and set time step to $\delta t=0.002$. We start with interfacial tensions $\sigma_{\mathrm{SS}}=$ $\sigma_{\mathrm{HS}}=\sigma_{\mathrm{HH}}=\sigma_{\mathrm{SP}}=\sigma_{\mathrm{HP}}=1.0$ at E14 stage, changing only the SC-HC tension linearly to $\sigma_{\mathrm{HS}}=0.6$, which qualitatively follows the change in adhesion strengths measured experimentally in terms of $\beta$-catenin intensities in auditory epithelium derived from WT mice at E16 stage (for methodology, see Section 4.2 ). Pattern formation at later stages after E16 cannot be explained only by adhesion. Indeed, the change in hair cell morphology from square to circular is thought to be caused by pronounced cytoskeletal dynamics within hair cells, leading to increased hair cell stiffness. To express this factor in our model, we evolve the interfacial tensions so that $\sigma_{\mathrm{SS}}<\sigma_{\mathrm{HS}}<\sigma_{\mathrm{HH}}$. Specifically, we impose their linear change reaching $\sigma_{\mathrm{SS}}=0.3, \sigma_{\mathrm{HS}}=0.6$, $\sigma_{\mathrm{HH}}=1.0, \sigma_{\mathrm{SP}}=0.65$ and $\sigma_{\mathrm{HP}}=1.0$ at E18 stage. Figure 5c and Movie S4 show that the expected patterns from E14 to E18 stages can be reproduced solely by designing the interfacial tensions. Although the nature of the relationship between interfacial tension and adhesion, contractility, stiffness, internal pressure, etc., is still unknown, our results show the significant potential of the level set-based approach as an effective tool for investigating this relationship.

Let us further test the proposed method by investigating pattern formation in auditory epithelium of Nectin-3 
KO mice, where the strong adhesion between HCs and SCs is inhibited at E16 stage. Based on experimental measurements (see Section 4.2, this is reflected in the model by setting $\sigma_{\mathrm{HS}}=0.9$ at E16, while all the remaining values of interfacial tensions are retained from the above WT simulation. The numerical results presented in Figure 5 $5 \mathrm{~d}$ and Movie S4 show that checkerboard pattern is not formed at E16 and the final pattern is similar to the pattern observed in Nectin-3 KO mice (Figure 5 5 ). These simulations indicate the importance of transitory formation of checkerboard pattern at the E16 stage for robust attainment of mosaic distribution of hair and supporting cells. In Supplementary Section I we demonstrate that results of simulation may depend on initial condition but this dependence is eliminated by including fluctuations in the model.
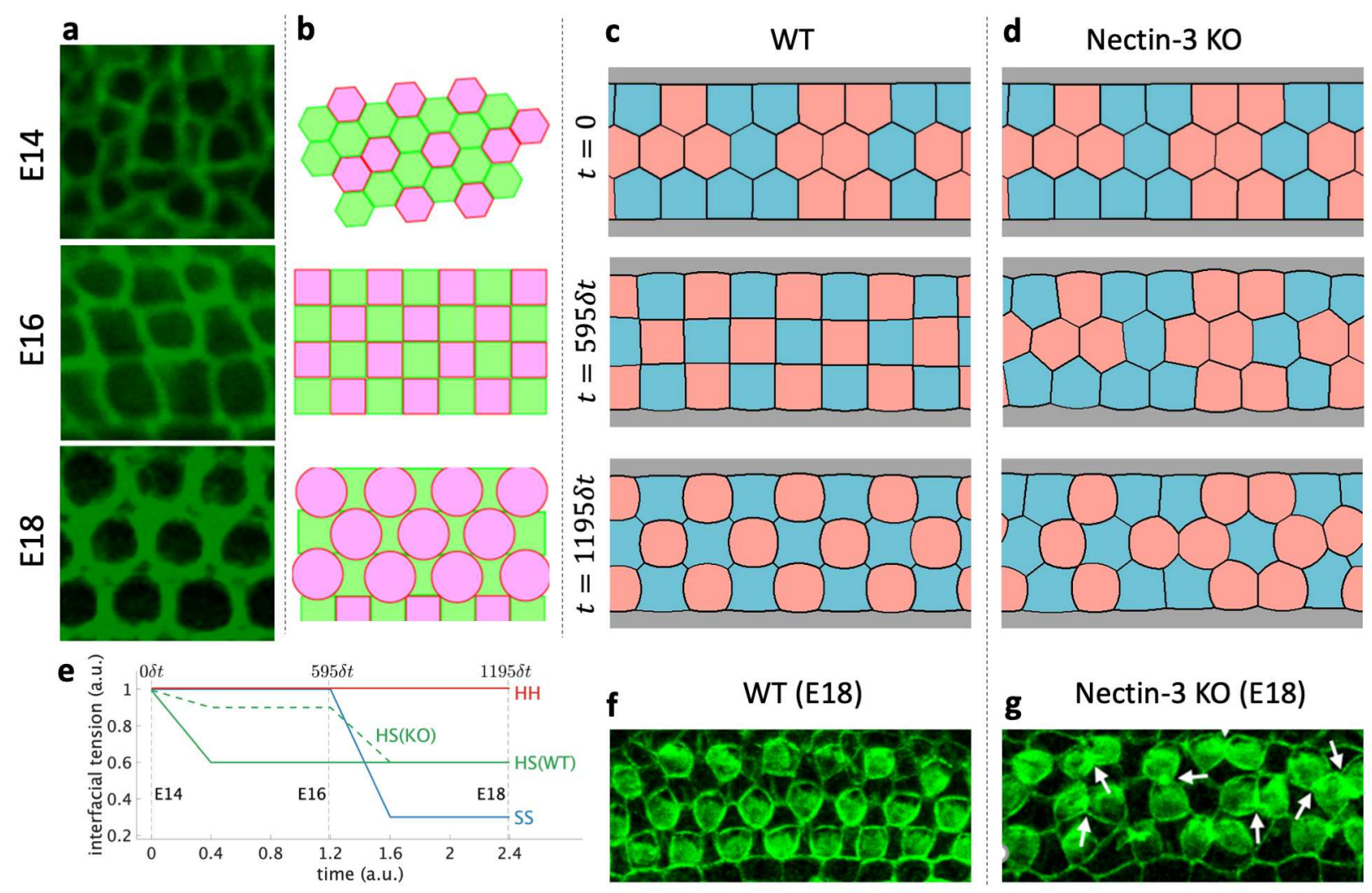

Figure 5: Actual images and simulation results of a developing auditory epithelium. a, Localization of junctional marker (ZO-1) at the apical surface of the auditory epithelium at embryonic days E14, E16, and E18. b, Schema of the distribution of hair cells (HC, pink) and supporting cells (SC, light green) observed in experiments (a and b: (C)2016 Togashi, originally published in Front. Cell Dev. Biol.). c-d, Simulations of cellular rearrangements of WT (c) and Nectin-3 KO (d) embryonic auditory epithelium from initial aggregate of 12 HCs (red), 12 SCs (blue), and one gray pillar cell, generated employing level set-based model. e, Plot of evolution of interfacial tensions at SS, HS, and HH junctions used for the simulations in $\mathbf{c}$ and $\mathbf{d}$. $\mathbf{f}-\mathbf{g}$, Actual images of auditory epithelium of WT mouse and Nectin-3 KO mouse at E18 stage, with arrows indicating aberrantly attached HCs (C)2011 Togashi et al., originally published in Science). 
The only publication on mathematical modeling of pattern formation in sensory epithelia that we are aware of, is the recent paper by Cohen et al. (2020). The authors used vertex dynamics model to replicate the checkerboard-like pattern in auditory epithelium, corresponding to Figure 5a-c (E16). Their main assumptions are global shear motion of sensory hair cells and repulsion force between hair cells, in addition to tension and volume preservation. However, although repulsive forces between hair cells play a central role in their model, there is no real evidence for the existence of such forces. On the other hand, our method, assuming only forces due to interfacial tension and volume preservation, reproduces the final pattern shown in Figure 5. (E18), which cannot be obtained using vertex dynamics model due to restrictions on available cell shapes. Our work indicates that differential interfacial tension is the main driving force of pattern formation in auditory epithelium. We believe that this result has a significant impact in developmental biology since a number of researchers expect that intercalation of cells in auditory epithelium requires a convergent extension process whose mechanism is not yet understood (Yamamoto et al. 2009).

\section{Discussion}

We have presented a mathematical model together with a computational method for simulating cellular rearrangements occurring in tissue morphogenesis, based on the level set approach. In particular, we adopt an implicit representation of cell-cell junctions and approximate their evolution by a thresholding scheme, which features good compatibility with the level set representation and a solid mathematical background including stability and convergence. We have combined this approach with auction dynamics algorithm to control cell volumes and augmented the numerical scheme with several aspects pertinent to cell biology, most importantly, a localization step which prevents cells from unnatural splitting during their rearrangement.

Because our level set-based approach is simply a numerical implementation of mathematical models founded on free energy minimization, it is therefore necessary to evaluate its pros and cons relative to its known counterparts (e.g., vertex dynamics model), and to specify types of problems, for which it is suitable. We have shown that the new level set-based algorithm enjoys the following merits: (1) it is able to accurately express complex geometries of cell-cell junctions and correctly realize cell contact angles, which is indispensable for finding the correct energy minimum and the corresponding dynamics including topology changes such as cell intercalations; (2) it has minimal number of parameters which is essential in using the model as a tool for testing biological hypotheses. In this respect, the proposed computational method surpasses its counterparts, but at the price of a more complex algorithm and moderate computational cost (see Supplementary Section F). Thus, it is recommended that one employs vertex dynamics for large-scale problems where detailed understanding of intercalation dynamics is not a priority, while level set-based method is more suitable for relatively small-scale simulations involving frequent 
topology changes and complex geometry.

A morphogenetic phenomenon, whose understanding essentially relies on these merits, is the formation of cellular patterns in sensory epithelia. Applying our level set-based method to the analysis of its underlying mechanism led us to the new discovery that differential interfacial tension plays a decisive role in the formation of sensory epithelia. Indeed, by simulating the epithelial formation based solely on interfacial tensions, we were able to reproduce the evolution of cellular patterns that is observed in experiments. Such finding was not possible until now because established methods either are not able to capture complex curved shapes of cells with significantly differing sizes and/or are not sufficiently mathematically accurate to grasp the frequent and delicate topology changes, such as intercalations, that are at the core of the patterning process.

\section{Methods}

\subsection{Numerical Scheme}

We present our algorithm based on the level set approach, starting with the formulation of the mathematical model. We represent an aggregate of cells as a bounded domain $\Omega \subset \mathbb{R}^{d}(d=2$ or 3$)$ partitioned into $N$ closed sets $\mathcal{C}_{1}, \ldots, \mathcal{C}_{N}$, representing cells (see Figure 1 a for the basic notation). It naturally follows that cell-cell junction $\gamma_{i j}:=\mathcal{C}_{i} \cap \mathcal{C}_{j}=\partial \mathcal{C}_{i} \cap \partial \mathcal{C}_{j}$ is the common boundary of sets $\mathcal{C}_{i}$ and $\mathcal{C}_{j}$.

We consider cellular rearrangement as the $L^{2}$-gradient flow (see Laux and Otto $(2016)$ and references therein for a precise definition) of the weighted surface energy

$$
E\left(\mathcal{C}_{1}, \ldots, \mathcal{C}_{N}\right)=\sum_{i \neq j} \sigma_{i j} \operatorname{Area}\left(\gamma_{i j}\right)
$$

constrained by each cell's prescribed volume $V_{\ell}^{0}(\ell=1,2, \ldots, N)$. Here, Area $\left(\gamma_{i j}\right)$ denotes the area of cell-cell junction $\gamma_{i j}$ in a $3 \mathrm{~d}$ model or the length of the junction in a $2 \mathrm{~d}$ model, and the weights $\sigma_{i j}=\sigma_{j i}>0$ for $i \neq j$ may be related to cell-cell adhesion and/or cell contractility (see Supplementary Section B for more). When $i=j$, we formally set $\sigma_{i j}=\sigma_{i i}=0$.

In materials science, this problem - considered without volume constraint to begin with - is widely known as the Mullins model for normal grain growth, where $\mathcal{C}_{i}$ denotes a grain in polycrystalline materials. To realize the least energy, lower semicontinuity of the functional is required, a necessary and sufficient condition for which is the triangle inequality $\sigma_{i k} \leq \sigma_{i j}+\sigma_{j k}$ for any distinct $i, j$ and $k$ (Morgan, 1997). The grain boundary $\gamma_{i j}$ in the $L^{2}$-gradient flow moves with a velocity $\mu_{i j} \sigma_{i j} \kappa_{i j} \boldsymbol{\eta}_{i j}$ where $\kappa_{i j}, \mu_{i j}$ and $\boldsymbol{\eta}_{i j}$ denote the mean curvature, mobility, and unit normal of $\gamma_{i j}$, respectively. Moreover, at triple junction where three grains $\mathcal{C}_{i}, \mathcal{C}_{j}$, and $\mathcal{C}_{k}$ meet, the Herring angle condition holds, that is, $\sigma_{i j} \boldsymbol{\eta}_{i j}+\sigma_{j k} \boldsymbol{\eta}_{j k}+\sigma_{i k} \boldsymbol{\eta}_{i k}=0$. Thus, grain boundaries in the annealing of pure metals (with equal surface tensions) evolve by mean curvature flow, where triple junctions meet at angles of $120^{\circ}$. 
Although the mathematical model is simple, its numerical realization is not at all obvious. Let us first explain the background of the proposed numerical scheme. Earlier works in simulating the above-mentioned grain boundary motion involve front-tracking (Bronsard and Wetton, 1995), which discretizes grain boundaries into finite number of points at which the mean curvature is explicitly calculated to determine its position at next time step. This resembles the vertex dynamics model, in the sense that both approaches evolve vertices based on explicitly calculated quantities. Consequently, its major drawback lies in its inability to handle grain boundaries that cross or have complicated topologies. Proper approximation of the subsequent evolution then requires some form of ad hoc "numerical surgery" which may lack physical justification and can be impractical to implement, particularly in three dimensions. To alleviate this drawback, Merriman et al. (1994) introduced the MBO thresholding scheme for diffusion-generated curvature-dependent motion of multiple junctions, which is based on the level set formulation of Osher and Sethian (1988) for propagating fronts with curvature-dependent speed. This scheme tracks interfaces implicitly by following level sets, facilitating natural handling of topological changes. In recent years, Esedoglu and Otto (2015) extended the MBO method to realize motion of grain boundaries in polycrystalline materials with arbitrary surface tensions. This method inherits the main advantages of the MBO approach: efficiency in the sense of low computational cost; and under a mild condition on the weights $\sigma_{i j}$, gradient stability in the sense that in every time step energy (1) is decreased.

With the aforementioned advantages, we introduce a level set-based algorithm to simulate cell dynamics in tissue morphogenesis. It is based on the Esedoḡlu-Otto algorithm but incorporates cell volume constraints and other aspects typical for cells. Following the lines in Esedoglu and Otto (2015), we briefly explain the derivation of the algorithm for the gradient descent of energy (1) under the volume constraints

$$
\operatorname{Volume}\left(\mathcal{C}_{\ell}\right)=V_{\ell}^{0}, \quad \ell=1, \ldots, N
$$

The area (corresponds to "length" in a two-dimensional model) of a cell-cell junction $\gamma_{i j}$ can be estimated using the so-called "heat content approximation", which states that the area of the junction is proportional to the heat that flows from cell $\mathcal{C}_{j}$ to cell $\mathcal{C}_{i}$ in a short time $\delta t$ :

$$
\operatorname{Area}\left(\gamma_{i j}\right) \approx \frac{1}{\sqrt{\delta t}} \int \chi_{\mathcal{C}_{i}} G_{\delta t} * \chi_{\mathcal{C}_{j}} d x
$$

Here $G_{\delta t}(x)=(4 \pi \delta t)^{-\frac{d}{2}} e^{-\frac{|x|^{2}}{4 \delta t}}$ is the $d$-dimensional Gaussian kernel, where $d$ is the spatial dimension of the model, and $\chi_{\mathcal{C}}$ denotes the characteristic function of a cell region $\mathcal{C}$. Hence, multiplying by the weights $\sigma_{i j}$ and adding over all junctions, the energy $E$ can, with a small error, be replaced by

$$
E\left(\mathcal{C}_{1}, \ldots, \mathcal{C}_{N}\right) \approx E_{\delta t}(\boldsymbol{u}):=\frac{1}{\sqrt{\delta t}} \sum_{i, j=1}^{N} \sigma_{i j} \int u_{i} G_{\delta t} * u_{j} d x
$$

where we have expressed the cell regions $\mathcal{C}_{\ell}, \ell=1, \ldots, N$ by a vector-valued function $\boldsymbol{u}=\left(u_{1}, \ldots, u_{N}\right)$ on $\Omega$, whose components $u_{\ell}(x)$ can take only two values: 1 if the point $x$ belongs to $\mathcal{C}_{\ell}$, or 0 if it does not. It 
was shown that $E_{\delta t}$ is a correct approximation of the original energy $E$ in the sense that it $\Gamma$-converges to $E$ when $\delta t \rightarrow 0$ (Appendix of Esedoḡlu and Otto (2015)).

Due to the condition on cell volumes, function $\boldsymbol{u}$ is constrained to the set

$$
\mathcal{B}:=\left\{\boldsymbol{u} \in\{0,1\}^{N}: \sum_{j=1}^{N} u_{j}(x)=1 \text { a.e. } x \in \Omega \text { and } \int_{\Omega} u_{\ell}=V_{\ell}^{0}, \ell=1, \ldots, N\right\},
$$

which is not convex. This poses a difficulty in the minimization problem, but it can be shown in a similar fashion to Esedoḡlu and Otto (2015) that the minimum of $E_{\delta t}$ over $\mathcal{B}$ coincides with the minimum over the convex set $\mathcal{K}$ obtained from $\mathcal{B}$ by relaxation, i.e., by allowing the components of $\boldsymbol{u}$ to take any value between 0 and 1 :

$$
\mathcal{K}:=\left\{\boldsymbol{u} \in[0,1]^{N}: \sum_{j=1}^{N} u_{j}(x)=1 \text { a.e. } x \in \Omega \text { and } \int_{\Omega} u_{\ell}=V_{\ell}^{0}, \ell=1, \ldots, N\right\} \text {. }
$$

The approximate energy $E_{\delta t}$ is still nonlinear, so to devise a simple minimization scheme, we adopt an iterative process by virtue of Lemma 5.2 in Esedoḡlu and Otto (2015) as follows. Given an approximation $\boldsymbol{u}^{k}$ of the minimizer of $E_{\delta t}$ in $\mathcal{K}$, we compute the next best approximation $\boldsymbol{u}^{k+1}$ by linearizing energy $E_{\delta t}(\boldsymbol{u})$ around $\boldsymbol{u}^{k}$ and defining $\boldsymbol{u}^{k+1}$ to be the minimizer of the linearized energy over $\mathcal{K}$ :

$$
\boldsymbol{u}^{k+1}=\arg \min _{\boldsymbol{u} \in \mathcal{K}} \mathcal{L}_{E_{\delta t}}\left(\boldsymbol{u} ; \boldsymbol{u}^{k}\right)
$$

Here $\mathcal{L}_{E_{\delta t}}$ is the linearized energy given by

$$
\mathcal{L}_{E_{\delta t}}\left(\boldsymbol{u} ; \boldsymbol{u}^{k}\right)=\frac{2}{\sqrt{\delta t}} \sum_{i=1}^{N} \int \varphi_{i}^{k} u_{i} d x, \quad \varphi_{i}^{k}:=\sum_{j=1}^{N} \sigma_{i j} G_{\delta t} * u_{j}^{k} .
$$

In the main algorithm below, we use function $\psi_{i}^{k}=1-\varphi_{i}^{k}$ instead of $\varphi_{i}^{k}$ for the purpose of reformulating the minimization problem into a maximization one. It was proved in Esedoḡlu and Otto (2015) and Laux and Otto (2016) that in the absence of volume constraints the sequence $\left\{\boldsymbol{u}^{k}\right\}$ decreases the approximate energy $E_{\delta t}$ with increasing step number $k$ and correctly approximates the $L^{2}$-gradient flow of the original energy $E$ in the limit $\delta t \rightarrow 0$.

In the case when there is no volume constraint, minimization (6) becomes a problem of minimizing a linear function over a simplex set $\mathcal{K}$. Thus the solution is obtained immediately as

$$
u_{i}^{k+1}(x)= \begin{cases}1 & \text { if } \varphi_{i}^{k}(x)=\min _{j} \varphi_{j}^{k}(x) \\ 0 & \text { otherwise }\end{cases}
$$

which leads to a very simple thresholding scheme. However, when the set $\mathcal{K}$ includes volume constraints, the solution of the minimization (6) involves unknown Lagrange multipliers $\lambda_{i j}$ :

$$
u_{i}^{k+1}(x)= \begin{cases}1 & \text { if } \varphi_{i}^{k}(x)=\min _{j}\left(\varphi_{j}^{k}(x)+\lambda_{i j}\right) \\ 0 & \text { otherwise }\end{cases}
$$


Direct computation of the Lagrange multipliers for more than 3 cells is complicated and can be avoided by the application of auction algorithm (Jacobs et al. 2018). The idea is to discretize the domain $\Omega$ into a uniform grid of points $\omega_{M}=\left\{x_{m}\right\}_{m=1}^{M} \subset \Omega$ and assign cell membership to each point of $\omega_{M}$ by simulating an auction, so that in the end each cell $\mathcal{C}_{\ell}$ contains $v_{\ell}^{0}$ points. Here, the number of grid points $v_{\ell}^{0}$ corresponds to the volume of the cell $\mathcal{C}_{\ell}$ in the sense of $v_{\ell}^{0} / M \approx V_{\ell}^{0} /|\Omega|$. It is natural to take the grid nodes $\left\{x_{m}\right\}$ identical to the grid nodes of the mesh used to numerically realize the convolutions in (7). The starting point of the auction dynamics algorithm is the configuration of the cells obtained by the gradient flow of energy without any volume constraint which is determined by the functions $\varphi_{i}^{k}$ above, or by the functions $\psi_{i}^{k}$ in the Algorithm below. In this configuration, some cells expand and some deflate with respect to their original volume. The algorithm then starts with all grid points unassigned and in arbitrary order takes the yet unassigned points and assigns them to their most preferred cell, where the extent of preference is in the beginning determined solely by the unconstrained configuration but later have to be adjusted via two new variables, namely the grid point's bid and the cell's price. This is because the "popular" cells, i.e., those which tend to expand in the unconstrained motion, become full in the sense that they reach the upper limit of points that can be accepted in them due to the volume constraint, and thus either some points have to be kicked out of the cell or the current point has to be assigned to its second favorite cell. The design of bids and prices that direct the unassigning and accepting of points resembles an auction performed by the grid points on the cells, leading to the naming of the algorithm. It is proved that the kicking out and accepting process finishes in a finite number of cycles and leads to the exact solution (at the discrete level of grid points) of the volume-constrained minimization problem. We refer to the main Algorithm below for precise description of the steps and to Jacobs et al. (2018) for a more concise exposition and rigorous proofs.

The Esedoḡlu-Otto scheme is simple and efficient but there are some issues that need to be tackled, in particular, the phenomena of wetting and nucleation (Esedoḡlu and Otto, 2015). Failure to satisfy the $\sigma$-triangle inequality condition leads to wetting, where a new cell $\mathcal{C}_{n}$ suddenly appears along an unrelated cell-cell junction $\gamma_{i j}$. Moreover, even when $\sigma$-triangle inequality is satisfied, a new cell may still get nucleated at a tricellular junction. For evolutions computed with auction dynamics, such wetting and nucleation will force a cell to split into two or more disjoint parts, some of which transfer to the wetting or nucleation regions. It is important to address this issue since such cell splitting phenomena do not occur during cellular rearrangements; yet it is possible that $\sigma_{i j}$ 's may not necessarily satisfy the triangle inequality condition in real tissues (see Supplementary Section C for more detailed exposition). To this end, we modify the auction algorithm by incorporating a topological constraint, so as to preserve cell connectivity. This makes sense physically, since individual cells only move in response to their local surroundings, i.e., to their neighboring cells. Hence, when we establish cell membership, we only allow local bidding processes in the auction, as shown in the following main algorithm (see Figure $1 \mathrm{~b}$ for illustrations). 
Algorithm (for numerical approximation of the $L^{2}$-gradient flow of energy (1) with preservation of cell volumes and connectivity)

Notation: Denote by $\chi_{\mathcal{C}}$ the characteristic function of a set $\mathcal{C}$. For given grid points $\omega_{M}=\left\{x_{m}\right\}_{m=1}^{M} \subset \Omega$ and a cell region $\mathcal{C}$, define the number of grid points in $\mathcal{C}$ by $|\mathcal{C}|:=\#\left\{m: x_{m} \in \mathcal{C}\right\}$.

Initialization: Split the time interval $[0, T]$ into $K$ subintervals of equal length $\delta t=T / K$. Discretize the computational domain $\Omega$ into a uniform finite grid $\omega_{M}=\left\{x_{m}\right\}_{m=1}^{M} \subset \Omega$. Prescribe initial cell regions $\left\{\mathcal{C}_{n}^{0}\right\}_{n=1}^{N}$ by assigning each discrete point $x_{m} \in \omega_{M}$ to a cell region. For each $n=1, \ldots, N$, record $v_{n}^{0}=\left|\mathcal{C}_{n}^{0}\right|$, the number of grid points in $\mathcal{C}_{n}^{0}$. Set the weights $\left\{\sigma_{i j}^{k}\right\}_{i, j=1, \ldots, N, k=0, \ldots, K-1}$ (here index $k$ refers to time) and the initialization parameter $0<\varepsilon \ll 1$ for the auction algorithm.

Algorithm: For each time step $k=0,1, \ldots, K-1$ perform the following steps, in order to determine the numerical cell regions $\left\{\mathcal{C}_{n}^{k+1}\right\}_{n=1}^{N}$ at time $t=(k+1) \delta t$ :

1. Solving heat equation. For each $x_{m} \in \omega_{M}$ compute

$$
\psi_{i}^{k}\left(x_{m}\right):=1-\sum_{j=1}^{N} \sigma_{i j}^{k}\left(G_{\delta t} * \chi_{\mathcal{C}_{j}^{k}}\right)\left(x_{m}\right),
$$

where $G_{\delta t}(x)=(4 \pi \delta t)^{-\frac{d}{2}} e^{-\frac{|x|^{2}}{4 \delta t}}$ is the $d$-dimensional Gaussian kernel.

2. Localized auction dynamics. Initialize prices $p_{n}=0$ and cell regions $\mathcal{C}_{n}^{k+1}=\emptyset(n=1, \ldots, N)$.

Until all points in $\omega_{M}$ are assigned, do:

(a) Find a point $x_{m} \in \omega_{M}$ which is not assigned.

(b) Let $i$ be the index of the cell to which $x_{m}$ belonged at step $k$.

(c) Find the set of the indices of neighboring cells of $\mathcal{C}_{i}^{k}: \mathcal{N}_{x_{m}}:=\left\{j: \partial \mathcal{C}_{i}^{k} \cap \partial \mathcal{C}_{j}^{k} \neq \emptyset\right\}$.

(d) Determine

$$
i^{*}=\arg \max _{i \in \mathcal{N}_{x_{m}}}\left(\psi_{i}^{k}\left(x_{m}\right)-p_{i}\right), \quad i^{\sharp}=\underset{i \in \mathcal{N}_{x_{m}} \backslash\left\{i^{*}\right\}}{\arg \max }\left(\psi_{i}^{k}\left(x_{m}\right)-p_{i}\right) .
$$

(e) If $\left|\mathcal{C}_{i^{*}}^{k+1}\right|=v_{i^{*}}^{0}$,

- find $l$ such that $x_{l}=\operatorname{argmin}_{x \in \mathcal{C}_{i^{*}}^{k+1}} b(x)$, and

- unassign $x_{l}$ from $\mathcal{C}_{i^{*}}^{k+1}$.

(f) Assign $x_{m}$ to $\mathcal{C}_{i^{*}}^{k+1}$.

(g) Calculate the bid $b\left(x_{m}\right)=p_{i^{*}}+\varepsilon+\left(\psi_{i^{*}}^{k}\left(x_{m}\right)-p_{i^{*}}\right)-\left(\psi_{i^{\sharp}}^{k}\left(x_{m}\right)-p_{i^{\sharp}}\right)$.

(h) Update the price $p_{i^{*}}=\min _{x \in \mathcal{C}_{i^{*}}^{k+1}} b(x)$.

It can be shown that thanks to the positive value of $\varepsilon$, the second auction dynamics step finishes in finitely many steps, yielding a partition of $\omega_{M}$ into cell regions, such that each cell region has the prescribed number of grid points (Jacobs et al., 2018). Characteristic functions of this partition are fed into the first step of 
the algorithm and the algorithm repeats until the final time is reached. The bid function $b$ appears for the first time in step 2(e) of the algorithm without being previously initialized but this definition is consistent since the bid has meaning only for grid points that are already assigned to a cell region. The bid expresses the extent to which the grid point "wants to be a member" of the cell region which it currently belongs to, and its role is to resolve conflicts among grid points that are trying to become members of the same, already full cell region. Movie S5 shows an animation of the auction dynamics algorithm for one time step of rearrangement in a simple 3-cell aggregate, tracking current cell price, node bid and nodal assignments.

We now briefly comment on the parameters related to the numerical implementation. The discretization parameters are the number $M$ of discrete points in the computational domain $\Omega$ and the time step size $\delta t$. The convolutions 10 in each step are efficiently computed on rectangular grids using fast Fourier transform (FFT) algorithm with a complexity of $O(M \log M)$ operations (Esedoglu and Otto, 2015). The time step can be changed throughout the computation but we emphasize that there are restrictions on the relative size of the spatial and temporal grids in order to obtain reasonable results; namely, an excessively small time step relative to the space grid size leads to incorrect stagnation of moving level sets. A common practice is to take $\delta t$ proportional to the first power of the spatial grid size $\delta x$ (Misiats and Yip, 2016). Moreover, the parameter $\varepsilon$ of the auction algorithm is taken as a small positive value and has the role of preventing a "price war" infinite loop, where the prices $p_{i}$ get stuck at a certain value. Too small $\varepsilon$ may result in an increase in computational time, while a large value may lead to deviations from the prescribed cell volumes. An idea of $\varepsilon$-scaling introduced in Jacobs et al. (2018) consists in starting with a relatively large $\varepsilon$ and repeating the auction with smaller and smaller values of $\varepsilon$, which not only eliminates the influence of this parameter but also improves both computational time and accuracy. The complexity of the auction step for a fixed $\varepsilon$ is $O(N v(\log v+N) C / \varepsilon)$, where $v=\max _{i} v_{i}^{0}$ and $C=\max _{i, x} \psi_{i}^{k}(x)$ (Jacobs et al. 2018). In summary, except for the unavoidable discretization parameters $M$ and $\delta t$, the output of the algorithm depends solely on the model's physically meaningful parameters $\sigma_{i j}$. The meaning and choice of these parameters are discussed in Supplementary Section B.

Regarding the choice of boundary conditions we remark that the Esedoḡlu-Otto scheme is originally formulated in the whole space $\mathbb{R}^{d}$ and thus numerical implementation on a bounded domain requires additional modifications. The simplest approach, which is usually compatible with biological settings, is to adopt a rectangular computational domain and apply Fourier transform to solve the convolutions (10). This naturally leads to periodic boundary condition for the evolution. Application of Fourier transform also allows for boundary conditions of Neumann type but other types of boundary conditions may require nontrivial adjustments and conceding the effective FFT method in favor of more general but more costly algorithms.

Next, we summarize basic mathematical properties of the algorithm, i.e., its stability and convergence. 
Firstly, for the original algorithm without volume constraint, Esedoglu and Otto (2015) showed that it is unconditionally gradient stable: for any choice of the time step $\delta t$, it dissipates in every time step the approximate energy (4) (which $\Gamma$-converges to energy (1)) under the sufficient condition that the surface tension matrix $\left\{\sigma_{i j}\right\}_{i, j=1}^{N}$ is conditionally negative semi-definite:

$$
\sum_{i, j=1}^{N} \sigma_{i j} \xi_{i} \xi_{j} \leq 0 \quad \text { for any }\left(\xi_{1}, \ldots \xi_{N}\right) \in \mathbb{R}^{N} \text { such that } \sum_{i=1}^{N} \xi_{i}=0 .
$$

This condition is often satisfied in materials science but there is no guarantee that it will hold in biological settings, e.g., cell-cell adhesiveness strengths in olfactory epithelium measured in terms of its $\beta$-catenin intensity values (Katsunuma et al., 2016). In such a case, it is possible to devise a slightly more complex version of the algorithm that guarantees gradient stability solely under the $\sigma$-triangle inequality condition (we refer to Section 5.4 of Esedoğlu and Otto (2015) for details). The convergence of the algorithm to the weak solution of the $L^{2}$-gradient flow of energy (1) has been proved in Laux and Otto (2016). We note that due to the fundamental idea of the algorithm to propagate interfaces over a fixed grid and due to the stagnation phenomenon mentioned above, the order of convergence is restricted to at most 1 in both time and space, while the order near multiple junctions turns out to be only $\frac{1}{2}$ in time.

The nontrivial difficulty in the analysis of the volume-preserving combined algorithm lies in the fact that the auction algorithm is in essence space-discrete, while all existing proofs deal with space-continuous problems. Moreover, in our scheme we localize the auction step which makes the analysis even more involved. However, the stability of the volume-constrained problem in the space-continuous setting (formulated using Lagrange multipliers for each cell's volume) can be proved in the same way and under the same assumptions as in the unconstrained case (see Xu et al. (2017) for the idea of the proof), while convergence has been established in Laux and Swartz (2017). This, together with the known convergence of the auction algorithm (at the spatially discretized level, the optimal solution can be achieved precisely) supports the expectation for the correct behavior of the combined scheme, which still remains to be precisely proved. Since a rigorous proof is beyond the scope of the paper, we have included a series of numerical tests confirming the correct behavior of our scheme. An exhaustive account on the results of these numerical tests is provided in Supplementary Section D with the following conclusions. The scheme is of order one in time away from junctions and the order of convergence falls to around 0.5 when triple junctions are involved. This was tested also on a configuration involving topology change. However, this convergence property holds true only for time steps larger than a certain threshold depending on the spatial grid - if the time step $\delta t$ is significantly smaller than the grid size $\delta x$ then interfaces stagnate and the scheme fails to converge. Further, the algorithm was tested on a three-phase configuration with triple junctions leading to an anisotropic double bubble and it was confirmed that its output closely follows an accurate front-tracking approximation of the evolution, with error decreasing with refinement of discretization. The same holds true for the stationary solution of double bubble evolution. 


\subsection{Quantification of Junctional Intensity of Mouse Sensory Epithelium}

Measurements of $\beta$-catenin intensity of cell-cell junctions in the mouse olfactory and auditory epithelium were performed by quantifying the pictures of specimens using ZEN software (Carl Zeiss). Quantifications were performed for normalized fluorescence intensities, and the average was calculated. $\alpha \mathrm{N}$-catenin KO mice and nectin-3 KO mice were generated as previously described (Togashi et al., 2002, Inagaki et al., 2005). The animal experiments were approved by the Institutional Animal Care and Use Committee and carried out according to the Kobe University Animal Experimental Regulations.

Correspondence and requests for materials should be addressed to Karel Svadlenka (karel@math.kyotou.ac.jp).

\section{Acknowledgements}

This work was supported by the Japan Society for the Promotion of Sciences through its Grants-in-Aid for Scientific Research (KAKENHI) [Grant Numbers 19K03634, 19H04965, 18H04764, 18H05481, 18K06219, 18H01139, 20H01823] and Grant-in-aid for JSPS Fellows [Grant Number 18F18016]; and the Japan Science and Technology Agency through its PRESTO (Precursory Research for Embryonic Science and Technology) Program [Grant Number JPMJPR1946]. The authors also thank to Dr. Sayaka Katsunuma for data sampling, and to Mr. Adrien Rey for his cooperation on the development of the numerical code during his short-term exchange student stay at Kyoto University in 2018.

\section{Data availability statement}

All data generated or analyzed during this study are included in this published article (and its supplementary information files) or are fully accessible in the cited previous work.

Accession numbers for cDNAs are listed as below: mouse nectin-1 gene, AF297665.1; mouse nectin-3 gene, NM_021495.4; mouse cadherin-1 gene, NM_009864.3; mouse cadherin-2 gene, NM_007664.5.

\section{Code availability}

All codes used to obtain the level set-based simulation data (doi: 10.24433/CO.7605938.v1) and to conduct numerical tests (doi: 10.24433/CO.9092704.v1) presented in this article are fully available on Code Ocean. 


\section{Author contributions}

Rhudaina Z. Mohammad: Methodology, Software, Formal analysis, Investigation, Writing original draft, Visualization. Hideki Murakawa: Conceptualization, Methodology, Writing, Review and editing. Karel Svadlenka: Methodology, Formal analysis, Writing, Review and editing, Supervision. Hideru Togashi: Validation, Investigation, Resources, Data curation.

\section{Competing interests}

The authors declare no competing interests.

\section{References}

Armstrong, N.J., Painter, K.J., Sherratt, J.A., 2006. A continuum approach to modelling cell-cell adhesion. J. Theor. Biol. 243, 98-113. doi $10.1016 / j \cdot j$ tbi.2006.05.030

Brakke, K.A., 2013. Surface Evolver. URL: http://facstaff.susqu.edu/b/brakke/evolver/evolver. html.

Brodland, G.W., 2002. The differential interfacial tension hypothesis (DITH): A comprehensive theory for the self-rearrangement of embryonic cells and tissues. Journal of Biomechanical Engineering 124, 188-197. doi $10.1115 / 1.1449491$

Brodland, G.W., 2004. Computational modeling of cell sorting, tissue engulfment, and related phenomena: A review. Applied Mechanics Reviews 57, 47-76. doi 10.1115/1.1583758.

Brodland, G.W., Chen, H.H., 2000. The mechanics of heterotypic cell aggregates: Insights from computer simulations. Journal of Biomechanical Engineering 122, 402-407. doi 10.1115/1.1288205

Bronsard, L., Wetton, B.T.R., 1995. A numerical method for tracking curve networks moving with curvature motion. Journal of Computational Physics 120, 66-87. doi $10.1006 /$ jcph.1995.1149.

Carrillo, J.A., Murakawa, H., Sato, M., Togashi, H., Trush, O., 2019. A population dynamics model of cellcell adhesion incorporating population pressure and density saturation. Journal of Theoretical Biology 474, 14-24. doi:10.1016/j.jtbi.2019.04.023

Chen, H.H., Brodland, G.W., 2000. Cell-level finite element studies of viscous cells in planar aggregates. Journal of Biomechanical Engineering 122, 394-401. doi:10.1115/1.1286563. 
Cohen, R., Amir-Zilberstein, L., Hersch, M., Woland, S., Loza, O., Taiber, S., Matsuzaki, F., Bergmann, S., Avraham, K.B., Sprinzak, D., 2020. Mechanical forces drive ordered patterning of hair cells in the mammalian inner ear. Nature Communications 11. doi 10.1038/s41467-020-18894-8.

Esedoḡlu, S., Otto, F., 2015. Threshold dynamics for networks with arbitrary surface tensions. Communications on Pure and Applied Mathematics 68, 808-864. doi 10.1002/cpa.21527.

Fletcher, A.G., Osborne, J.M., Maini, P.K., Gavaghan, D.J., 2013. Implementing vertex dynamics models of cell populations in biology within a consistent computational framework. Progress in Biophysics and Molecular Biology 113, 299-326. doi 10.1016/j.pbiomolbio.2013.09.003

Foty, R.A., Steinberg, M.S., 2005. The differential adhesion hypothesis: a direct evaluation. Developmental Biology 278, 255-263. doi:10.1016/j.ydbio.2004.11.012.

Glazier, J.A., Graner, F., 1993. Simulation of the differential adhesion driven rearrangement of biological cells. Phys. Rev. E 47, 2128-2154. doi 10.1103/PhysRevE.47.2128.

Harris, A.K., 1976. Is cell sorting caused by differences in the work of intercellular adhesion? A critique of the Steinberg hypothesis. Journal of Theoretical Biology 61, 267-285. doi:10.1016/0022-5193(76)90019-9

Inagaki, M., Irie, K., Ishizaki, H., Tanaka-Okamoto, M., Morimoto, K., Inoue, E., Ohtsuka, T., Miyoshi, J., Takai, Y., 2005. Roles of cell-adhesion molecules nectin 1 and nectin 3 in ciliary body development. Development 132, 1525-1537. doi:10.1242/dev.01697.

Ishimoto, Y., Morishita, Y., 2014. Bubbly vertex dynamics: A dynamical and geometrical model for epithelial tissues with curved cell shapes. Physial Review E 90, 052711. doi 10.1103/PhysRevE.90.052711.

Jacobs, M., Merkurjev, E., Esedoḡlu, S., 2018. Auction dynamics: A volume constrained MBO scheme. J. Comput. Physics 354, 288-310. doi $10.1016 / j \cdot j c p .2017 .10 .036$

Katsunuma, S., Honda, H., Shinoda, T., Ishimoto, Y., Miyata, T., Kiyonari, H., Abe, T., Nibu, K.I., Takai, Y., Togashi, H., 2016. Synergistic action of nectins and cadherins generates the mosaic cellular pattern of the olfactory epithelium. The Journal of cell biology 212, 561-575. doi $10.1083 /$ jcb. 201509020.

Laux, T., Otto, F., 2016. Convergence of the thresholding scheme for multi-phase mean-curvature flow. Calculus of Variations and Partial Differential Equations 55, 129. doi 10.1007/s00526-016-1053-0.

Laux, T., Swartz, D., 2017. Convergence of thresholding schemes incorporating bulk effects. Interfaces and Free Boundaries 19, 273-304. doi $10.4171 /$ IFB/383.

Maître, J.L., Heisenberg, C.P., 2011. The role of adhesion energy in controlling cell-cell contacts. Current Opinion in Cell Biology 23, 508-514. doi $10.1016 / j \cdot c e b .2011 .07 .004$ 
Maître, J.L., Niwayama, R., Turlier, H., Nédélec, F., Hiiragi, T., 2015. Pulsatile cell-autonomous contractility drives compaction in the mouse embryo. Nature Cell Biology 17, 849-855. doi:10.1038/ncb3185.

Maître, J.L., Turlier, H., Illukkumbura, R., Eismann, B., Niwayama, R., Nédélec, F., Hiiragi, T., 2016. Asymmetric division of contractile domains couples cell positioning and fate specification. Nature 536, 344-348. doi $10.1038 /$ nature18958.

Merriman, B., Bence, J.K., Osher, S.J., 1994. Motion of multiple junctions: A level set approach. Journal of Computational Physics 112, 334-363. doi 10.1006/jcph.1994.1105.

Misiats, O., Yip, N., 2016. Convergence of space-time discrete threshold dynamics to anisotropic motion by mean curvature. Discrete and Continuous Dynamical Systems - A 36, 6379-6411. doi:10.3934/dcds. 2016076 .

Morgan, F., 1997. Lowersemicontinuity of energy clusters. Proceedings of the Royal Society of Edinburgh: Section A Mathematics 127, 819-822. doi 10.1017/S0308210500023842.

Nagai, T., Honda, H., 2001. A dynamic cell model for the formation of epithelial tissues. Philosophical Magazine B 81, 699-719. doi:10.1080/13642810108205772.

Nose, A., Nagafuchi, A., Takeichi, M., 1988. Expressed recombinant cadherins mediate cell sorting in model systems. Cell 54, 993-1001. doi 10.1016/0092-8674(88)90114-6.

Osher, S., Sethian, J.A., 1988. Fronts propagating with curvature-dependent speed: Algorithms based on Hamilton-Jacobi formulations. Journal of Computational Physics 79, 12-49. doi:10.1016/0021-9991(88) 90002-2.

Salvador, T., Esedoḡlu, S., 2019. A simplified threshold dynamics algorithm for isotropic surface energies. Journal of Scientific Computing 79, 648-669. doi 10.1007/s10915-018-0866-8.

Steinberg, M.S., 1963. Reconstruction of tissues by dissociated cells. Science 141, 401-408. doi:10.1126/ science.141.3579.401.

Steinberg, M.S., Takeichi, M., 1994. Experimental specification of cell sorting, tissue spreading, and specific spatial patterning by quantitative differences in cadherin expression. Proceedings of the National Academy of Sciences of the United States of America 91, 206-209. doi 10.1073/pnas.91.1.206.

Togashi, H., 2016. Differential and cooperative cell adhesion regulates cellular pattern in sensory epithelia. Frontiers in cell and developmental biology 4:104. doi $10.3389 /$ fcell.2016.00104.

Togashi, H., Abe, K.A., Mizoguchi, A., Takaoka, K., Chisaka, O., Takeichi, M., 2002. Cadherin regulates dendritic spine morphogenesis. Neuron 35, 77-89. doi 10.1016/S0896-6273(02)00748-1. 
Togashi, H., Kominami, K., Waseda, M., Komura, H., Miyoshi, J., Takeichi, M., Takai, Y., 2011. Nectins establish a checkerboard-like cellular pattern in the auditory epithelium. Science 333, 1144-1147. doi-10. $1126 /$ science.1208467.

Xu, X., Wang, D., Wang, X.P., 2017. An efficient threshold dynamics method for wetting on rough surfaces. Journal of Computational Physics 330, 510-528. doi 10.1016/j.jcp.2016.11.008

Yamamoto, N., Okano, T., Ma, X., Adelstein, R.S., Kelley, M.W., 2009. Myosin ii regulates extension, growth and patterning in the mammalian cochlear duct. Development 136, 1977-1986. doi $10.1242 /$ dev.030718

Zhao, J., Cao, Y., DiPietro, L.A., Liang, J., 2017. Dynamic cellular finite-element method for modelling large-scale cell migration and proliferation under the control of mechanical and biochemical cues: a study of re-epithelialization. J. R. Soc. Interface 14, 20160959. doi 10.1098/rsif.2016.0959. 


\section{Supplementary Material for}

\section{A level set-based approach for modeling cellular rearrangements in tissue morphogenesis}

\section{Supplementary Section A. Overview of Numerical Methods}

Morphogenetic phenomena can be investigated at various scales ranging from molecular up to continuum level; see, e.g., Armstrong et al. (2006); Carrillo et al. (2019) for a successful reproduction of cellular patterns formed due to cell-cell adhesion and other factors at the continuum level. Meanwhile, our objective is to understand the mechanisms of interactions among cells on the microscopic level, and thus we need to precisely resolve shapes of individual cells. Numerical methods for free energy minimization at microscopic scale include, among others, vertex dynamics model, cellular Potts model, front-tracking, and finite element methods (Brodland, 2004), see Figure A.6. The main difference of these approaches lies in how cells are geometrically represented. Vertex dynamics model (Nagai and Honda, 2001; Fletcher et al., 2013) represents cells as polygons where mechanical forces are applied to vertices. In this model, polygonal vertices migrate so as to decrease an energy potential, which includes a penalty term to enforce each cell's preferred volume. Such geometric representation, however, cannot precisely approximate cell-cell junctions of complex shapes with nonzero curvature and tissues composed of cells with significantly differing sizes. This problem was addressed in Ishimoto and Morishita (2014), where curvature of the junctions is added as a new parameter of the geometry, leading to "bubbly vertex dynamics". Cellular Potts model (Glazier and Graner, 1993), on the other hand, discretizes the continuous cell aggregate configuration onto a fixed regular lattice; thereby, representing each cell as a number of grid points. At each grid point, one calculates how the energy (based on cell-cell adhesion, cell incompressibility, and chemotaxis) changes as the grid point transitions to a randomly selected neighbor. If this elicits a decrease in energy, the grid point is allowed to transition to its neighbor. As a result, when a cell moves, it may lose or gain some grid points on the lattice. Due to its local nature, this approach does not have the potential to accurately deal with topology changes triggered by a global energy balance. Lastly, finite element method (Chen and Brodland, 2000, Zhao et al., 2017) partitions polygonal cells into a finite number of elements to solve mechanical equations characterizing tissue dynamics. In the two-dimensional setting, each cell-cell junction is represented as a piecewise linear curve and interfacial tensions are modeled using constant force on point masses along each cell-cell junction. In this sense, this formulation acts like the vertex dynamics model. A similar approach is front-tracking, where junctions are approximated by discrete points, which are moved by the given evolution law. Brakke's Surface Evolver (Brakke, 2013) is the most developed and general program based on this approach that we are aware of. It can simulate various types of interface evolution, including multiphase evolutions in 3 dimensions but 
applications to complex cellular rearrangements including the analysis of its performance with respect to frequent topology changes, are still missing.

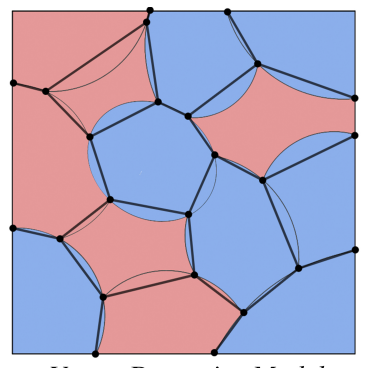

Vertex Dynamics Model

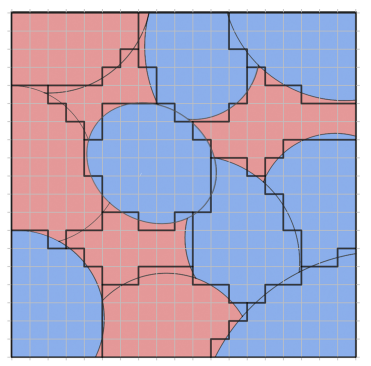

Cellular Potts Model

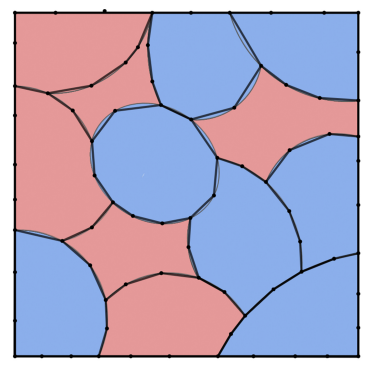

Front-tracking Method

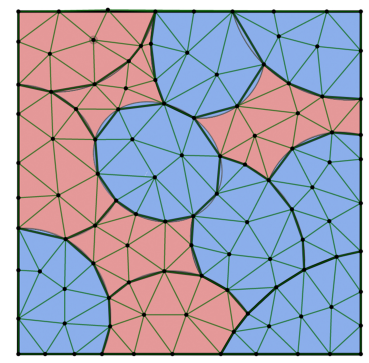

Finite Element Method

Figure A.6: Major numerical models for tissue morphogenesis. An aggregate of red and blue cells geometrically represented according to vertex dynamics model, cellular Potts model, front-tracking, and finite element method.

\section{Supplementary Section B. Model Parameters}

Here we elaborate on physically relevant parameters that are required or allowed by the model introduced in Methods. Besides parameters $M, \delta t$ and $\varepsilon$ used in the numerical implementation of the model, the only parameters of the model itself are the weights $\sigma_{i j}$, showing a major advantage over other similar models. For example, vertex dynamics model includes, besides these weights, several further parameters, such as the elastic stiffness coefficients for the volume and perimeter, minimal distance of two vertices to initiate T1 transformation, a parameter for the resulting distance of the vertices, etc. Consequently, our model allows us to focus on the role of the target force represented by the parameters $\sigma_{i j}$ without having to analyze the impact of other effects. This is essential as such analysis often presents an infeasible task.

The choice of specific values for $\sigma_{i j}$ 's depends on the purpose of the modeling, and usually requires novel ideas and insights into the modeled phenomenon. Moreover, these values act as a doorway through which experimental measurements can be reflected in the model, in a quantitative manner, and thus their choice is also closely tied to the design of experiments. In this sense, it is impossible to devise a general recipe, and we will only give some examples of possible choices for $\sigma_{i j}$ 's.

There are two basic competing approaches to the design of $\sigma_{i j}$, which, as we believe, are peacefully united in our model: Steinberg's differential adhesion hypothesis (Steinberg, 1963; Foty and Steinberg, 2005), and the differential interfacial tension hypothesis (DITH) put forward by Brodland (2002). Steinberg (1963) based his theory on the observation of similarities between liquid sorting and cell sorting, and postulated that cellular rearrangements can be in certain cases explained by the tendency of the cells to maximize their intermolecular adhesion. Evidences speaking for the correctness of the hypothesis were provided by several experiments, the prominent one being the establishment of a hierarchical sequence of segregation among 
several different tissues. Later, Brodland (2002) pointed out that adhesion acts in the opposite direction than interfacial tension, and formulated a more complete and precise hypothesis, giving it a new name. Discussions in the biological modeling community on the significance of these and other hypotheses have been conducted for several decades (Harris, 1976, Brodland, 2004). Our purpose here is not necessarily to probe into these discussions; but rather to present a general model that can encompass most of the approaches proposed up to date. For example, the model can take the weights $\sigma_{i j}$ to be the cortical tensions of the cell membrane (Maitre et al. 2015) (in which case there is a direct correspondence with the physical meaning of these parameters), or the weights may reflect the adhesion energy per area (Katsunuma et al., 2016; Maitre and Heisenberg, 2011) (in which case $\sigma_{i j}$ 's do not have the meaning of adhesion energy but depend on that energy in a suitable manner that has to be determined as a part of the particular model), or a combination of both. In Section 2.3 we give a specific example of the design of the weights $\sigma_{i j}$ that express the adhesion energy and are quantified through the experimentally measurable quantity of $\beta$-catenin intensity. On the other hand, a precise quantification of the relation between measured $\beta$-catenin intensities and magnitude of adhesion strength or of the corresponding interfacial tension is still a challenging task. Recently, several techniques have been proposed to quantify the strength of cadherin-dependent cell-cell adhesion, e.g., flow chamber assay, atomic force microscopy, and dual pipette assay; however, it seems difficult to apply these techniques to intact tissue. Here, we define cell-cell adhesion strength based on the measured value of $\beta$ catenin intensity from intact tissue, because $\beta$-catenin intensity is well known to be correlated with adhesive strength.

We would like to bring attention to the fact that the weights $\sigma_{i j}$ in the algorithm can be time-dependent, which is expressed by the index $k$ in equation 10 . This is essential, as almost all morphogenetic phenomena are driven by temporally changing forces. Moreover, this time-dependence turns our model from a mere energy minimizing gradient descent system into an out-of-equilibrium one, as expected for a model of a living system.

The minimal set of parameters, i.e., the weights $\sigma_{i j}$, can be augmented by new parameters according to necessity to express various additional aspects of the target biological phenomenon. One example of such additional parameters are the mobilities $\mu_{i j}$ mentioned in the beginning of Methods. In fact, the algorithm as presented in Section 2.1 advances each junction $\gamma_{i j}$ with the mobility $\mu_{i j}=1 / \sigma_{i j}$. If one wishes to prescribe mobilities in a different way, it is possible to modify the algorithm by introducing so-called retardation terms, as explained in Section 5.1 of Esedoḡlu and Otto (2015). This method was improved in Salvador and Esedoḡlu (2019) by replacing the computation of retardation functions by a more efficient convolution step.

Another optional set of parameters $\left\{a_{i}^{k}, b_{i}^{k}\right\}_{i=1}^{N}$ is related to the lower and upper bounds controlling the volumes of individual cell regions, thus allowing for certain cell compressibility, in the sense that we look for the energy minimizing configuration such that the discrete cell volumes $v_{i}^{k}$ (i.e., the number of grid points 
in cell $\mathcal{C}_{i}$ at a given time $t_{k}$ ) satisfy

$$
a_{i}^{k} \leq v_{i}^{k} \leq b_{i}^{k}, \quad i=1, \ldots, N .
$$

It is possible to modify the auction algorithm to extend it to this type of constraint. The implementation of the upper bound does not significantly change the algorithm but the lower bound requires running a reverse auction where cell regions bid on points (see Jacobs et al. (2018), Section 3.3). One can also incorporate random effects in the algorithm - either by randomly changing cell volumes (see Jacobs et al. (2018), Section 3.4 ) or by adding a suitable noise to the weights $\sigma_{i j}$ (see Supplementary Section I).

\section{Supplementary Section C. Topological Singularities}

Here we show that the localization scheme resolves the wetting and nucleation problem of the original Esedoḡlu-Otto algorithm. We consider a wetting case for a 4-cell aggregate of three types: 2 blue, 1 red, and 1 gray cell (see Figure C.7), with $\sigma_{\mathrm{BB}}=1.5, \sigma_{\mathrm{BG}}=0.5$, and $\sigma_{\mathrm{RR}}=\sigma_{\mathrm{GG}}=\sigma_{\mathrm{BR}}=\sigma_{\mathrm{RG}}=1.0$. Here, B, R, G denote the cells of type blue, red and gray, respectively, so that, for example, $\sigma_{\mathrm{BR}}$ means the interfacial energy weight for the junction between blue and red cell. Note that this violates the triangle inequality, since $\sigma_{\mathrm{B}_{1} \mathrm{G}}+\sigma_{\mathrm{B}_{2} \mathrm{G}}=1<1.5=\sigma_{\mathrm{B}_{1} \mathrm{~B}_{2}}$. We evolve the initial configuration using three different algorithms: the original Esedoglu and Otto (2015) scheme alone, the same scheme with standard auction dynamics (Jacobs et al., 2018), and finally with our proposed scheme employing a localized auction dynamics. Here, we discretize domain $\Omega=[0,1] \times[0,1]$ uniformly into $M=500 \times 500$ points, prescribe periodic conditions on its boundary, and set time step $\delta t=0.0005$. Results of the simulation are shown in Figure C.7 and Movie S6.

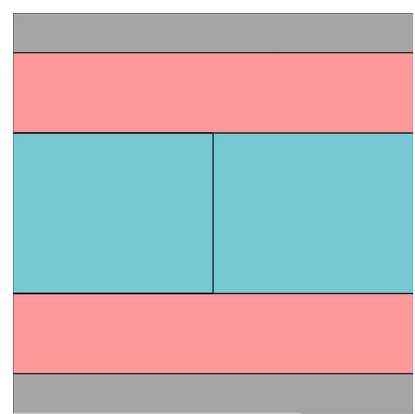

Initial configuration

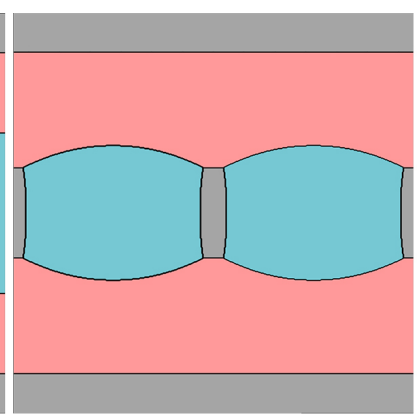

Esedoglu-Otto $(E O)$ scheme

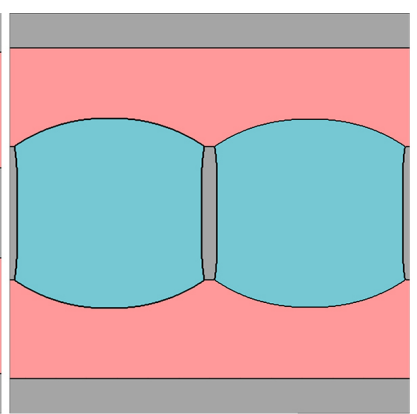

EO + auction dynamics

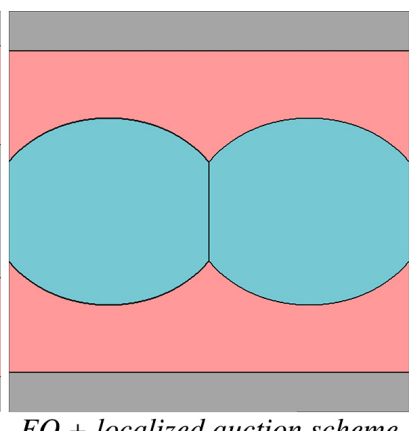

$E O+$ localized auction scheme

Figure C.7: Wetting phenomenon in the numerical implementation of level set-based methods. Initial 4-cell configuration and its evolution under a wetting condition due to violation of $\sigma$-triangle inequality at $t=50 \delta t$ using Esedoḡlu-Otto scheme (EO); EO scheme with auction dynamics algorithm; and EO scheme with localized auction dynamics.

We observe that for the Esedoḡlu-Otto scheme, a new gray cell grows at the BB-junctions - wetting occurs. 
When implemented with usual auction dynamics, we see that the gray cell splits and some of its parts appear in the BB-junction. In these cases, optimization (6) is taken over all possible cells, and thus $\sigma$ triangle inequality becomes important to rule out wetting. However, with the localized auction dynamics, cell splitting due to wetting is avoided. This is because, for points in the neighborhood of the BB-junction, maximization 11 is localized and only taken over the two blue cells. Hence, irregardless of whether $\sigma$ triangle inequality holds, wetting does not occur with localized auction dynamics.

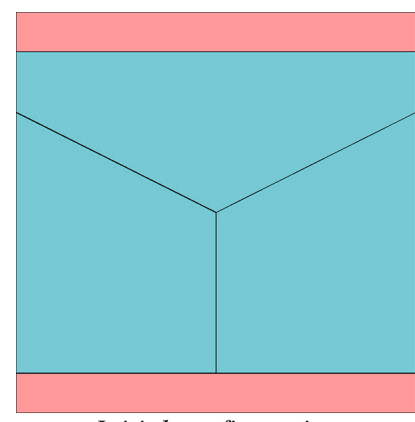

Initial configuration

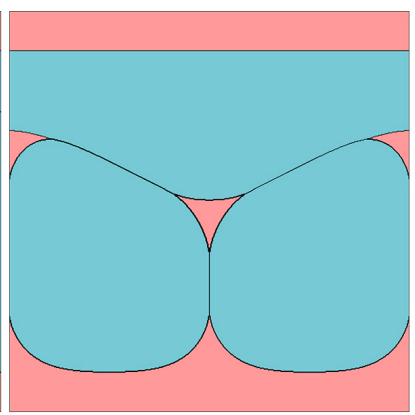

Esedoglu-Otto (EO) scheme

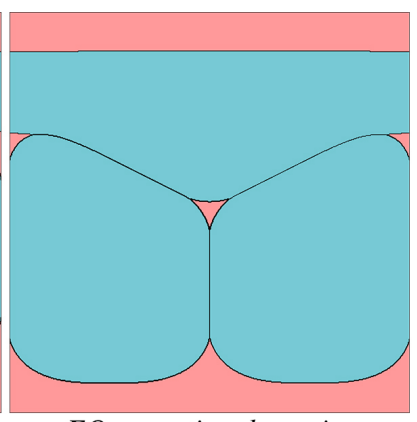

EO + auction dynamics

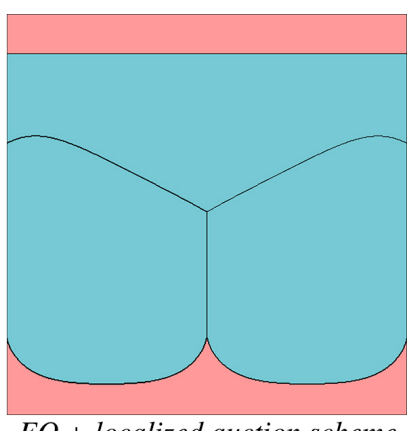

EO + localized auction scheme

Figure C.8: Nucleation phenomenon in the numerical implementation of level set-based methods. Initial 4 -cell configuration and its evolution at $t=10 \delta t$ using Esedoglu-Otto (EO) algorithm resulting in nucleation of red cell at the blue triple junction; EO scheme with auction dynamics where red cell splitting persists; and EO scheme with localized auction dynamics which preserves cell connectivity.

Next, we consider a nucleation case for a 4-cell aggregate of two types: 3 blue and 1 red cell (see Figure C.8) under the same conditions on the domain and time step size as in the first simulation. With $\sigma_{\mathrm{BB}}=\sigma_{\mathrm{RR}}=1.0$ and $\sigma_{\mathrm{BR}}=0.53$, we evolve the initial configuration using the same numerical schemes as above. Note that since the $\sigma_{i j}$ 's satisfy the triangle inequality condition, wetting cannot take place. However, Figure C.8 and Movie S7] show that in the evolution computed by Esedoglu-Otto algorithm alone, red cells grow in the vicinity of the blue tricellular junction - an unnatural cell dynamics. This phenomenon persists even when auction algorithm is incorporated, but is completely eliminated upon introducing the localization.

\section{Supplementary Section D. Numerical Tests}

In this section, we present a series of numerical tests demonstrating the properties of the newly proposed scheme. In order to avoid jump-like behavior of errors inherent to algorithms using characteristic functions, we implement a simple idea for obtaining a sub-grid spatial accuracy in all numerical tests used to measure some type of error in numerical solution. Namely, after the diffusion step, we calculate the intersections of level sets with grid lines, use this information to obtain the ratio of area that each phase occupies in every grid cell, and based on these ratios construct a piecewise linear upgrade of the characteristic function that captures the interface more precisely. Moreover, to accelerate the computation, we optimized the auction 
Table D.1: Relative errors of level set-based algorithm for varying mesh-time configurations

\begin{tabular}{|c|c|cc|cc|}
\hline \multirow{2}{*}{ mesh resolution } & \multirow{2}{*}{ number of time steps } & \multicolumn{2}{|c|}{ growing circle } & \multicolumn{2}{c|}{ shrinking circle } \\
\cline { 3 - 6 } & & relative error & order & relative error & order \\
\hline $100 \times 100$ & 15 & 0.0231424 & - & 0.1208982 & - \\
$200 \times 200$ & 30 & 0.0111530 & 1.0531 & 0.0517636 & 1.2238 \\
$400 \times 400$ & 60 & 0.0059719 & 0.9012 & 0.0259983 & 0.9935 \\
$800 \times 800$ & 120 & 0.0027456 & 1.1211 & 0.0115676 & 1.1683 \\
$1600 \times 1600$ & 240 & 0.0014114 & 0.9600 & 0.0061546 & 0.9104 \\
\hline
\end{tabular}

dynamics step with bid priority queuing.

\section{Supplementary Section D.1. Numerical Convergence Test}

We start with investigation of convergence order away from triple junctions. We consider the volumepreserving mean curvature flow of a three-phase initial configuration on a square domain $\Omega=[0,1] \times[0,1]$ with periodic boundary conditions, as shown in Figure D.9 , where both red and blue phases consist of two disjoint circles of radii $R=0.15$ and $r=0.12$. We set $\sigma_{\mathrm{BR}}=\sigma_{\mathrm{BG}}=\sigma_{\mathrm{RG}}=1.0$, which results in the larger circles growing as the smaller circles shrink and eventually vanish; thereby, satisfying the following system of differential equations:

$$
\frac{d r}{d t}=-\frac{1}{r}+\frac{2}{r+R}, \quad \frac{d R}{d t}=-\frac{1}{R}+\frac{2}{r+R}
$$

For various mesh-time configurations, we run our algorithm and compare its output with the precise approximation of the exact solution to the coupled differential equation (D.1) obtained by Runge-Kutta (RK4) method of order 4. We take the relative error of the radius of the resulting circles at around three-fourths of the extinction time of the smaller circles, that is, in our setup, at time $t=0.01875$, which is the final time of the simulation. Table D.1 shows that the order of convergence of our algorithm is linear (cf. Jacobs et al. (2018)). Figure D.9 b,c shows log-log plot of the absolute error of radii of the resulting circles at the same time $t=0.01875$. One observes that taking too small time step relative to spatial grid size leads to an increase in error due to unwanted stagnation of interfaces.

\section{Supplementary Section D.2. Stationary Anisotropic Double Bubble Test}

To analyze the long-time behavior of the scheme, we consider a three-phase initial configuration on a square domain $\Omega=[0,1] \times[0,1]$ with periodic boundary conditions, as shown in Figure D.10a where two phases are identical squares sharing one common side of length 0.35 . We denote $\mathcal{C}_{1}$ as the left square, $\mathcal{C}_{2}$ as the right 

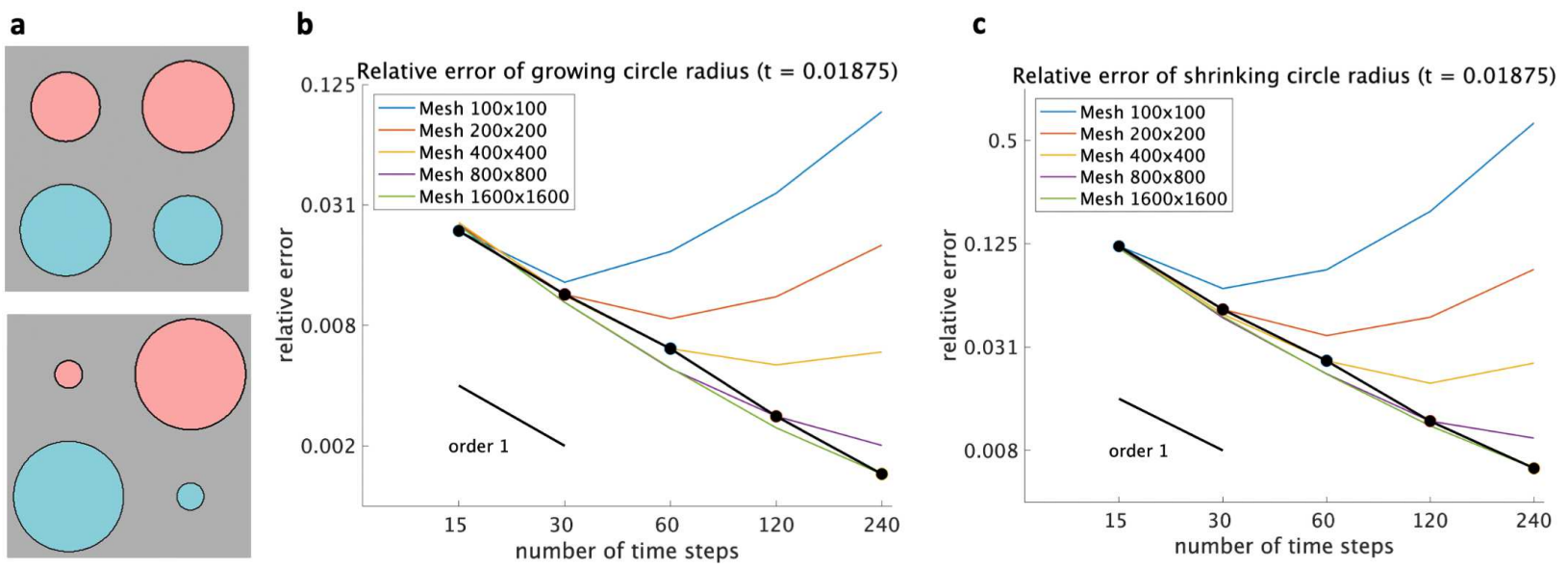

Figure D.9: Basic convergence test for level set-based algorithm. a, A three-phase initial configuration and its volume-preserving mean curvature evolution at time $t=35 \delta t$ with $\delta t=0.0008$. b,c, $\log$-log plot of relative error of mean radius of larger circles (b) and smaller circles (c) at $t=0.01875$ for various mesh-time configurations. Black points denote error values corresponding to space-time discretizations $(\delta x, \delta t)$ satisfying $\delta x=8 \delta t$ and demonstrate that the convergence order is linear.

square, and $\mathcal{C}_{0}$ as the remaining phase region, and set $\sigma_{01}=\frac{1}{2} \sqrt{3}, \sigma_{02}=1$, and $\sigma_{12}=\frac{1}{2}$. Under volumepreserving anisotropic mean curvature flow, the stationary solution of such configuration is an anisotropic double bubble, which consists of three circular arcs meetings at two junction points with corresponding contact angles $\theta_{0}=\frac{5 \pi}{6}, \theta_{1}=\frac{\pi}{2}$, and $\theta_{2}=\frac{2 \pi}{3}$.

Using our algorithm, we take numerical stationary solution at time $t=0.5$ for various mesh-time configurations and compare these with the exact stationary solution. Using a least-squares fitting method to fit the resulting interface points to a circle, we compute the relative error of the radius of the best fitted circle on each interface. Figure D.10 d shows the log-log plot of the relative error for each arc and mesh-time configuration, respectively. In addition, we check whether our numerical stationary solution satisfies the necessary angle conditions at the triple junction. We determine the numerical contact angles using the normal vector to the best fitted circles at each triple junction. The obtained errors are displayed in Figure D.10.

\section{Supplementary Section D.3. Contact Angle Analysis across Topological Change}

Here we look into the ability of the algorithm to realize correct contact angles at triple junctions undergoing topology change. We consider a 4-cell aggregate of two types: 2 red and 2 blue cells on a square domain $\Omega=[0,1] \times[0,1]$ with periodic boundary conditions (see Figure D.11 ). Taking $\sigma_{\mathrm{BB}}=\sigma_{\mathrm{RR}}=\sqrt{2}$ and $\sigma_{\mathrm{BR}}=1$ evolves the configuration in such a way that at both middle junctions, the contact angle opening towards a blue cell changes from the initial angle $180^{\circ}$ to $90^{\circ}$, until a topological change occurs, that is, the 

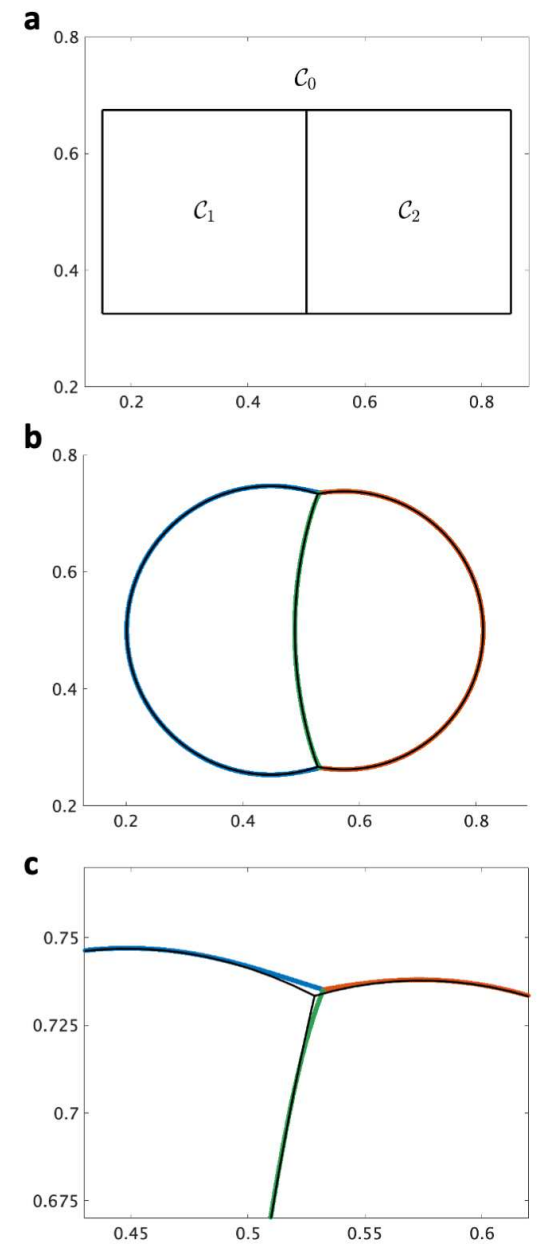
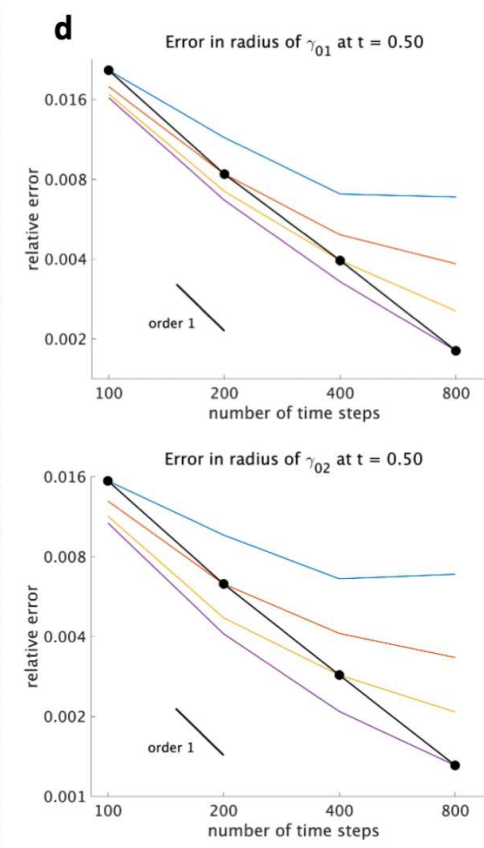

Error in radius of $\gamma_{12}$ at $\mathrm{t}=0.50$

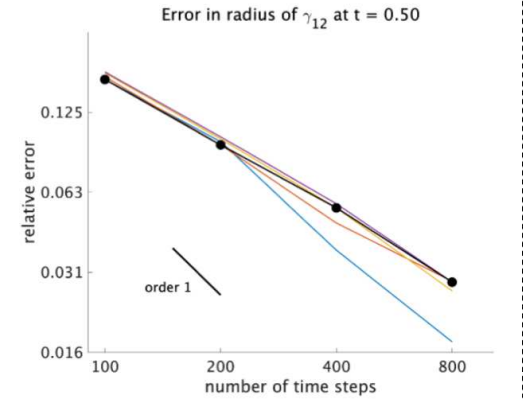

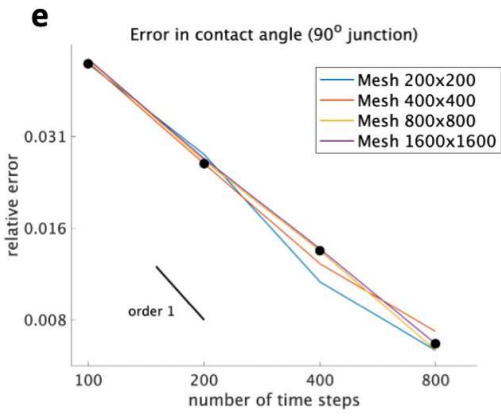
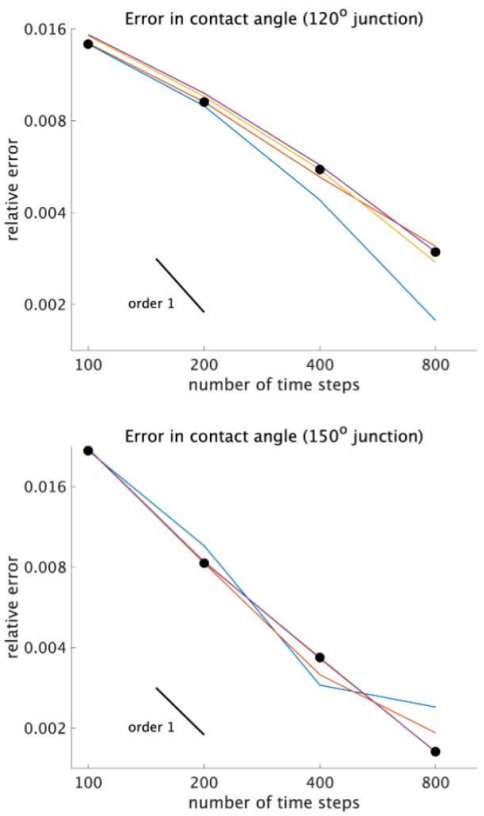

Figure D.10: Numerical results of stationary double bubble test a, Three-phase initial configuration; b, its stationary numerical solution (color) using our algorithm with $1600 \times 1600$ mesh at $t=0.5$ overlapping with the exact solution (black); and c, detail of the upper triple junction. d, Log-log plot of relative error in radius of the best fitted circle to interface $\gamma_{01}, \gamma_{02}$, and $\gamma_{12}$ for various mesh-time configurations. e, Log-log plot of relative error in contact angles at the top junction on phase $\mathcal{C}_{1}, \mathcal{C}_{2}$, and $\mathcal{C}_{0}$ for various mesh-time configurations. Black points denote error values obtained at space-time discretizations satisfying $\delta x=\delta t$, demonstrating convergence.

blue cells intercalate and the red cells split. At this point, the contact angle changes from $90^{\circ}$ to $135^{\circ}$ and the cell-cell junctions deform until they reach a stationary state.

We run our level set-based algorithm until time $t=0.2$ for various mesh-time configurations (see Table D.2. To measure the numerical contact angles, we find the best fitted circle to each interface (excluding the junction point) and compute the contact angles using their tangent lines. In particular, we take the portion of the interface whose distance away from the junction, falls on the interval $(0.005,0.10)$, as shown in Figure D.11]. We then compute the $L^{1}$ error on contact angles in the time interval from $t=0.05$ to $t=0.15$, and display the results in Table D.2 and Figure D.11. We see that error decreases as both mesh and time 
are further refined. Figure D.11 also shows that the numerical time when topological change occurs differs slightly for each mesh-time configuration.

Table D.2: $L^{1}$-error of contact angle measure for varying mesh-time configurations

\begin{tabular}{|c|c|cc|}
\hline \multirow{2}{*}{ mesh resolution } & number of time steps & \multicolumn{2}{|c|}{ contact angle } \\
\cline { 3 - 4 } & & $L^{1}$ error & order \\
\hline $200 \times 200$ & 100 & 0.0289831 & - \\
$400 \times 400$ & 200 & 0.0186973 & 0.6324 \\
$800 \times 800$ & 400 & 0.0088110 & 1.0855 \\
$1600 \times 1600$ & 800 & 0.0044431 & 0.9877 \\
\hline
\end{tabular}

a
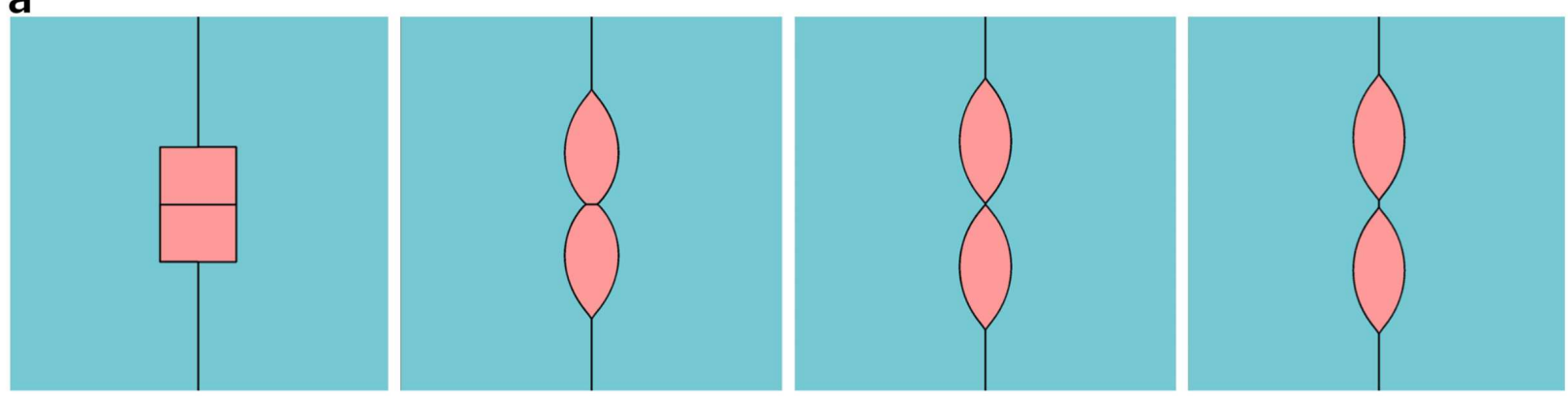

$\mathbf{b}_{0.7}$ mesh 800x800 t: 0.05 dist: $(0.005,0.100)$

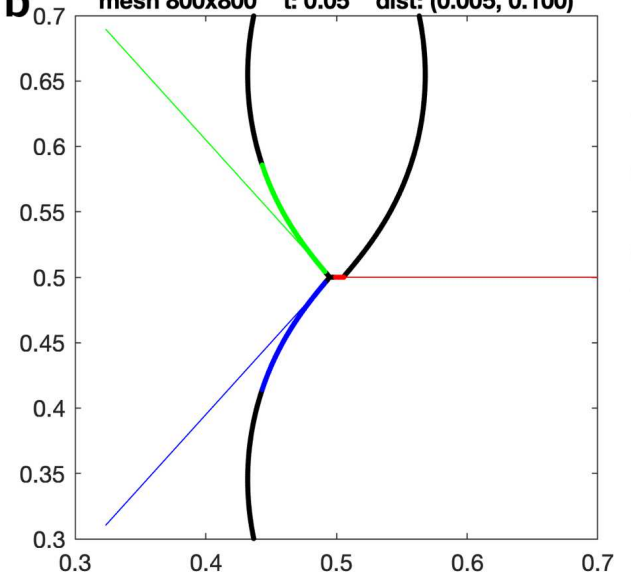

C

Contact angle measure over time

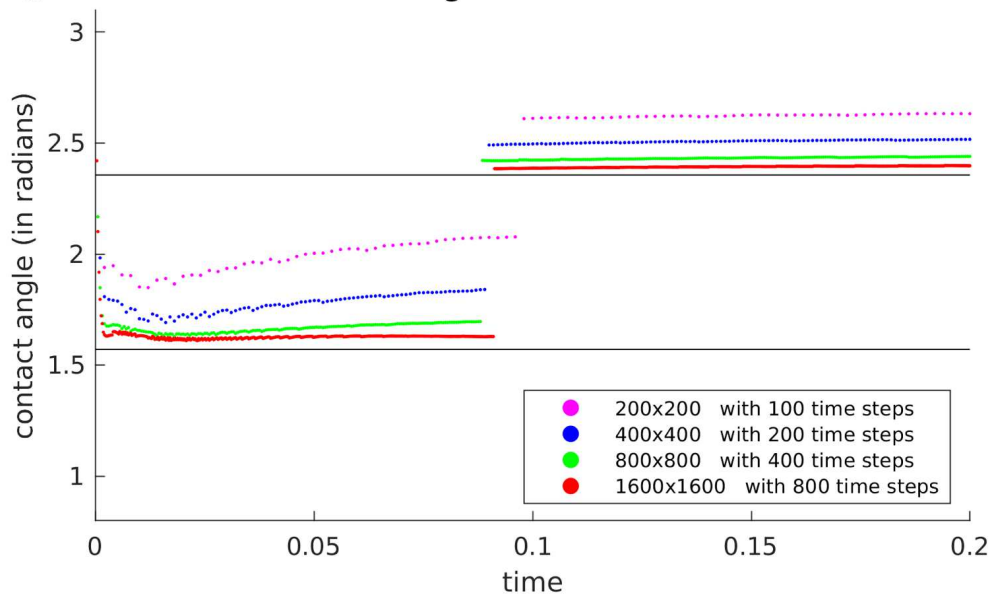

Figure D.11: Numerical test on topology change. a, (From left to right) An initial configuration of two cell types: 2 red and 2 blue cells, and its evolution using our algorithm with $\delta t=0.00025$ at times $t=100 \delta t, 400 \delta t$, and $800 \delta t$ on a $800 \times 800$ mesh. $\mathbf{b}$, The portion of the interface at time $t=0.05$, whose distance away from the junction falls on the interval $(0.005,0.10)$, used to calculate the contact angles for the $800 \times 800$ mesh. c, Evolution of the magnitude of contact angle measured as in $\mathbf{b}$ across topology change, shown for several mesh-time discretizations. Theoretically, the value should jump from $\pi / 2$ to $3 \pi / 4$ radians, as shown by the black lines. 
Using the same initial configuration as in Figure D.10 where two phases are identical squares sharing one common side of length 0.35 , we study how well our algorithm captures the dynamics of the evolution. Since analytical solution is not available, we implemented a front-tracking algorithm with automatic redistribution of points along interfaces that can deal also with triple junctions, and used its high-resolved solution at time $t=0.024$ as an accurate approximation of the true solution. At this time, the double bubble is powerfully evolving and far from the stationary state. Table D.3 and Figure D.12 show that the error, defined as the area of the symmetric set difference of the right bubble's front-tracking approximation and its realization by our algorithm, decreases with mesh-time grid refinement. However, due to the presence of triple points the overall convergence rate falls below linear.

Table D.3: Error of evolving double bubble vs front-tracking scheme
\begin{tabular}{|c|c|c|c|}
\hline mesh resolution & time step size & error & order \\
\hline $100 \times 100$ & 0.0080 & 0.006570 & - \\
$200 \times 200$ & 0.0040 & 0.004938 & 0.4118 \\
$400 \times 400$ & 0.0020 & 0.003843 & 0.3620 \\
$800 \times 800$ & 0.0010 & 0.003190 & 0.2685 \\
$1600 \times 1600$ & 0.0005 & 0.002782 & 0.1975 \\
\hline
\end{tabular}

a

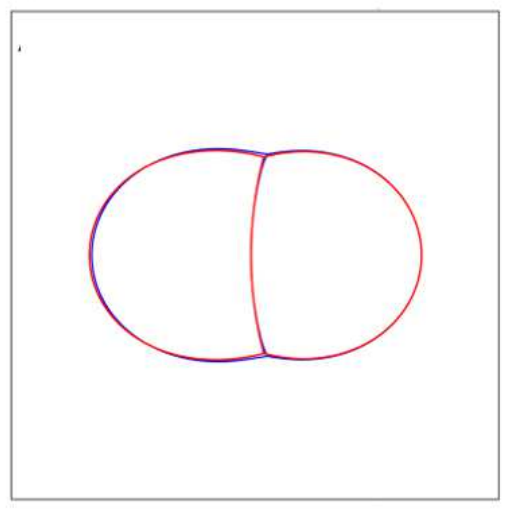

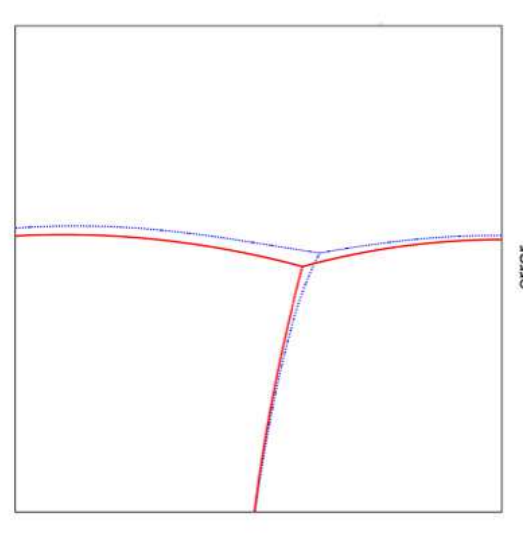

b

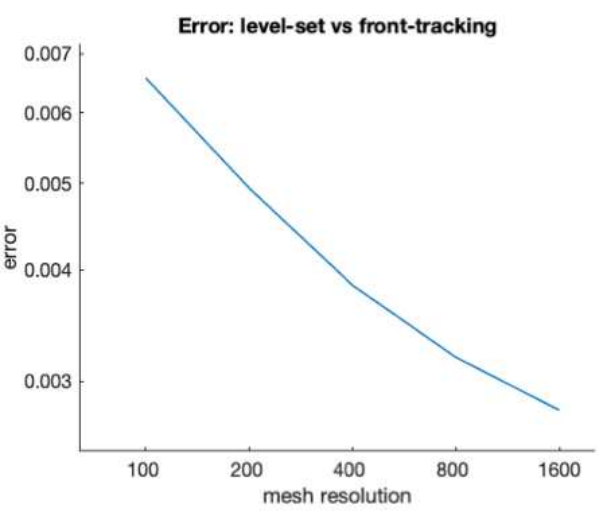

Figure D.12: Numerical test on multiphase dynamics. a, Plot of evolution of anisotropic double bubble using level set-based method (blue) and front-tracking method (red) at time $t=0.024$, along with a zoom-in on the upper triple junction. b, Log-log plot of the interfacial error vs front-tracking method for different mesh-time configurations from Table D.3. 

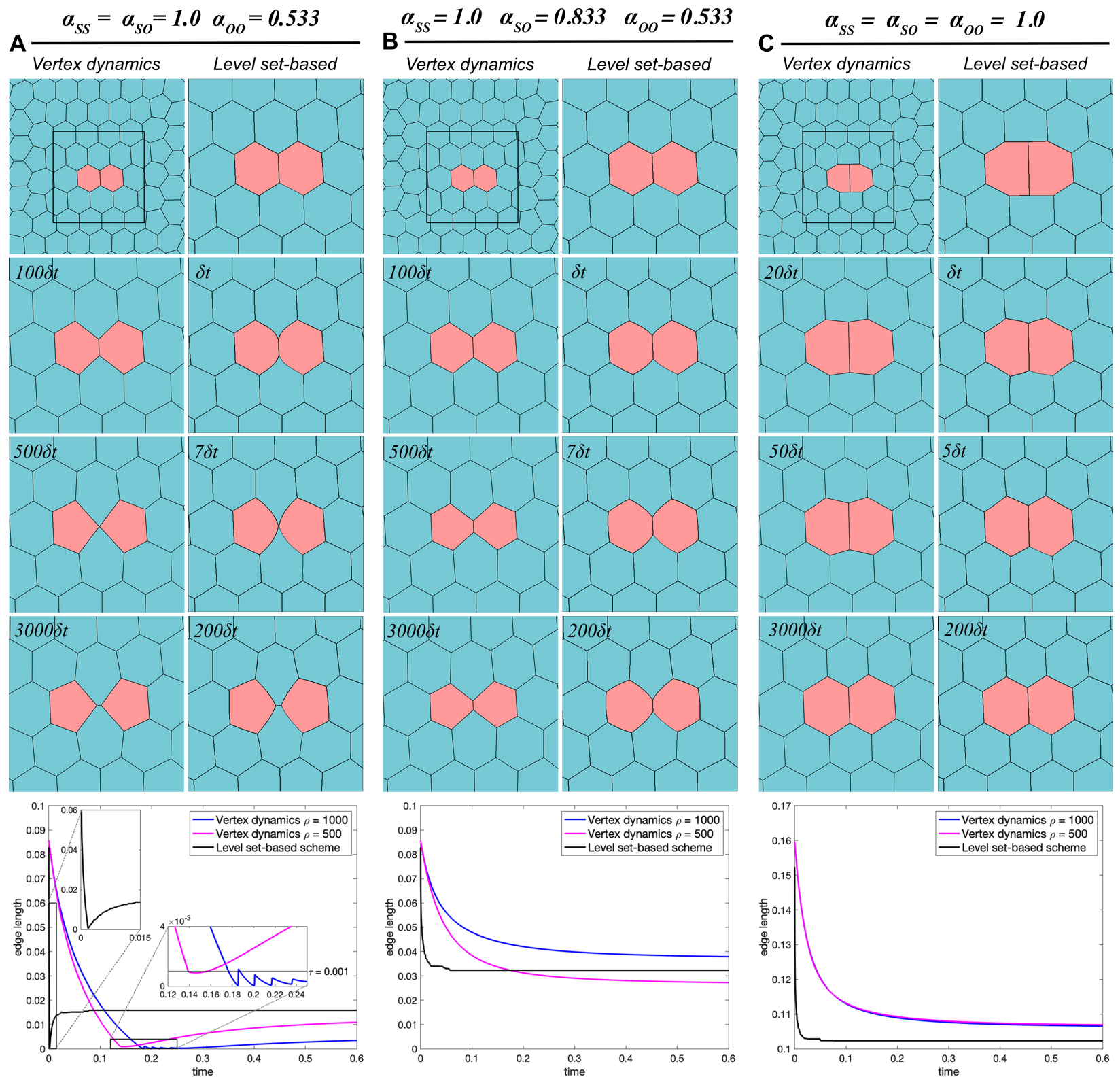

Figure E.13: Vertex dynamics vs. Level set-based model. Three test cases of adhesion strengths: $(\mathrm{A}) \alpha_{\mathrm{SS}}=$ $\alpha_{\mathrm{SO}}=1.0$ and $\alpha_{\mathrm{OO}}=0.533$ leading to cellular intercalation; (B) $\alpha_{\mathrm{SS}}=1.0, \alpha_{\mathrm{SO}}=0.833$ and $\alpha_{\mathrm{OO}}=0.533$ where no intercalation occurs; and (C) $\alpha_{\mathrm{SS}}=\alpha_{\mathrm{SO}}=\alpha_{\mathrm{OO}}=1.0$ which shortens OO-junction. Initial aggregate of 62 blue SCs and 2 red OCs (top left), its zoomed-in configuration (top right), and its evolution via vertex dynamics model with $\rho=500$ (left column) and level set-based approach (right column) for each test case. (Bottom) Plot showing the shrinkage of the OO-junction length (and the formation of a new SS-junction for the case where cellular intercalation occurs), generated using vertex dynamics model (blue and magenta, expressing different volume penalties $\rho$ ) and our level set-based approach (black) for each case. 


\section{Supplementary Section E. Comparison with Vertex Dynamics}

To highlight the ability of the algorithm to deal with topology changes, such as cell intercalations, let us delve into cellular pattern formations in developmental stages in the olfactory epithelium (OE). Katsunuma et al. (2016) hypothesized that heterophilic trans-interaction between nectin-2 on olfactory cells (OCs) and nectin-3 on supporting cells (SCs) promote recruitment in the cell-cell junction of the cadherin-catenin complex whose representative marker, $\beta$-catenin indicates the differential adhesions required to drive selforganized cell movements in OE. Following the differential adhesion hypothesis (Steinberg, 1963 Foty and Steinberg, 2005), Katsunuma et al. (2016) simulated two cases of cellular patterns: one with adhesion strengths $\alpha_{\mathrm{SS}}=\alpha_{\mathrm{SO}}=1.0$, and $\alpha_{\mathrm{OO}}=0.533$; while the other had a weaker adhesion $\alpha_{\mathrm{SO}}=0.833$. Using the vertex dynamics model with $\sigma_{i j}=\alpha_{i j}^{-1}$, they were able to confirm that the first case leads to cellular intercalation, while the second does not; thereby, supporting their idea that differential adhesion in heterotypic cell-cell junctions drives cell intercalations.

In order to compare the performance of the standard vertex dynamics algorithm and our scheme, we consider an initial cellular aggregate of $62 \mathrm{SCs}$ (blue) and 2 OCs (red) cells of almost equal volumes, similar to that in Katsunuma et al. (2016), with periodic boundary conditions on the square domain $\Omega=[0,1] \times[0,1]$ (see Figure E.13). We simulate both cases using the vertex dynamics model and our proposed scheme, with the same time step size $\delta t=0.0003$. For the vertex dynamics model, we employ the same potential as in (Katsunuma et al., 2016) with two types of cell volume penalty $(\rho=1000$ and $\rho=500$ ) and minimum threshold distances $\tau=10^{-3}$ for T1-transition. Moreover, for our level set-based approach, we discretize the domain uniformly into $M=1000 \times 1000$ points. Numerical results are shown in Figure E.13 - note here that the times where the snapshots are taken at largely different for each method (see also Movies S8, S9. and $\mathrm{S} 10]$.

We observe that both methods do lead to cellular intercalation when adhesion strengths $\alpha_{\mathrm{SS}}=\alpha_{\mathrm{SO}}=1.0$, and $\alpha_{\mathrm{OO}}=0.533$ are set. However, essential differences are found in the results. First, the time needed for the intercalation to occur in the vertex dynamics algorithm heavily depends on the minimum T1-transition threshold distance $\tau$, which can be seen by following the magenta line in Figure E.13A - the edge length starts to increase when this line hits the level $\tau$. In other words, a small change in $\tau$ may result in largely different times of intercalation, which in turn may have significant impact on the global dynamics of the aggregate. Second, and probably the most prominent difference between the two methods lies in the time rate of change of the junction length, i.e., the vertex dynamics motion is reluctant to undergo topology changes. Lastly, inspecting Figure E.13A, it is clear that both methods yield different final shapes of the intercalated cells. We remark that the jump-like behavior of the junction length in the level-set based method (black line), which can be observed in Figures E.13 and E.14 is due to fact that the level set method is performed on a fixed grid causing sudden changes of the length as a tricellular point jumps from one grid cell to another. On 
the other hand, the meshless vertex dynamics moves the tricellular points freely in space and hence yields smoothly changing lengths. However, the jumps observed in the level-set based method diminish with the refinement of the grid.
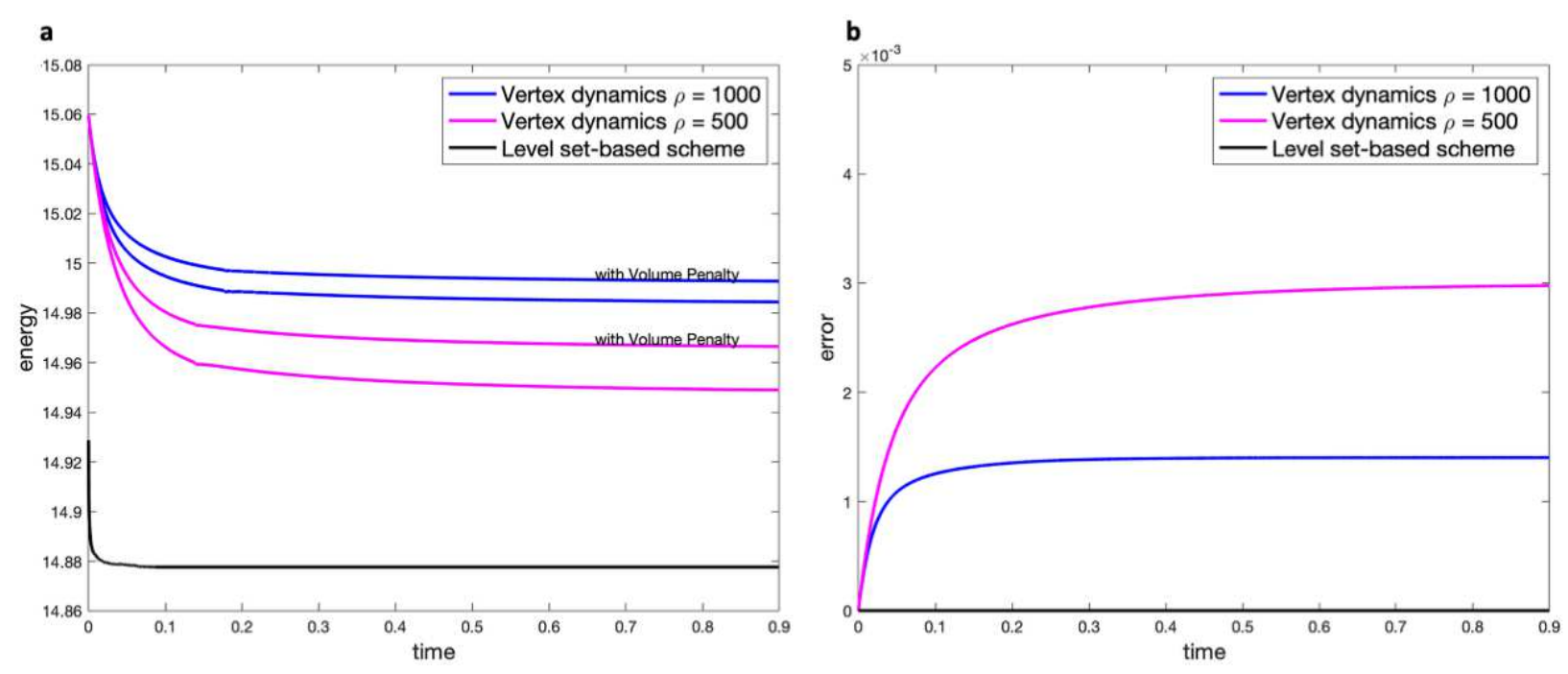

Figure E.14: Evolution of energy and cell volumes for the simulation in Figure E.13A. a, The total energy (upper blue curve) and its surface energy component (lower blue curve) of the vertex model for volume penalty $\rho=1000$ and analogous results for penalty $\rho=500$ (magenta). The black line shows the evolution of energy of level set-based method. The discrepancy in initial energy for both methods is caused by the different representation of cell shapes. $\mathbf{b}$, The evolution of error in cell volumes defined at time $t_{k}$ as the maximum absolute deviation of cell volumes from the prescribed volume, where the volume is calculated as polygonal area in the vertex dynamics model, and by $v_{i}^{k}(\delta x)^{2}$ in the level set based model.

To confirm whether this aspect of vertex dynamics is pertinent to topology changes such as intercalation, we have compared the evolution of junction lengths with cases where no intercalation occurs. Results for one such setting are shown in Figure E.13B , where the difference in adhesion strengths (having $\alpha_{\mathrm{SS}}=$ $\left.1.0, \alpha_{\mathrm{SO}}=0.833, \alpha_{\mathrm{OO}}=0.533\right)$ drives the OCs towards intercalation but is not strong enough for the intercalation to occur. Another test case, depicted in Figure E.13C sets all adhesion strengths equal to 1.0 and considers an initial configuration with unnaturally long junction between the red cells. One observes that the dynamics in this last case is similar for both methods leading to approximately the same stationary junction length, although the vertex dynamics model evolves slightly slower. In the "almost intercalating" test case of Figure E.13B, the vertex dynamics model lags behind the level set-based scheme and converges to a significantly different stationary length of the junction, which also strongly depends on the parameters of the vertex dynamics model, in this case, the volume penalty. Meanwhile, in all test cases, the level set-based algorithm swiftly approaches the steady state configuration, irregardless of the presence of intercalation, which conforms with its convergence proof of Laux and Otto (2016). From these findings, we may conclude that vertex dynamics model tends to slow down the evolution when topology changes are to take place, 
contrary to what is expected from the energy minimization principle.

This difference between the models is mainly caused by three factors, as follows. The first one is due to the different form of their energies, since the vertex model includes not only the surface energy but also other terms such as volume penalty term, etc. Secondly, the correct cell contact angles are not realized in the vertex dynamics model, particularly for cell-cell junctions that should be theoretically curved, which may alter succeeding cell dynamics. This, on the contrary, does not occur in the level set-based approach, as it is theoretically proved to satisfy the tricellular angle condition. Lastly, the vertex dynamics model can only take a restricted set of paths to minimize its energy. Indeed, since the level set-based method allows for arbitrary shapes of junctions, contrary to the vertex model where only polygonal shapes are allowed, it is able to follow the gradient descent of the energy correctly; while the vertex dynamics model is delayed (and for some cases, completely stopped) by having to take only polygonal deformations.

We elaborate on the first difference mentioned in the previous paragraph. In our model, the energy consists only of surface energy, and the volume preservation is realized through the constraint condition. Thus, the cell sizes are preserved precisely. On the other hand, in the vertex model, the energy is defined as the sum of surface energy and volume penalty term, while it is necessary to use a large value of penalty coefficient $\rho$ in order to preserve the cell sizes to some extent. The effect of the surface energy term is then relatively weakened when $\rho$ is large. In this way, the level set-based approach allows us to focus on the effect of surface or adhesion energy, unlike the vertex model where it is difficult to separate and correctly understand this effect (see Figure E.14 for a detailed analysis). This provides a typical example of the importance of reducing model parameters: the vertex model does not facilitate the understanding of the adhesion mechanism because the influence of its two additional parameters, namely the volume penalty coefficient $\rho$ and threshold distance for $\mathrm{T} 1$ transition $\tau$, cannot be easily analyzed. Indeed, as one can see in Figure E.13A, mere doubling of the volume penalty coefficient $\rho$ leads to a completely different behavior of the edge length. The oscillations of the edge length for the stronger volume penalty represent a tug of war triggered by the T1 transition between the elastic volume term and the surface adhesion term in the energy.

\section{Supplementary Section F. Computational Cost of the Algorithm}

Here we perform several numerical experiments to assess the computational cost of the level set-based algorithm and confront it with a different representative method, namely the vertex dynamics. Contrary to the vertex dynamics, where the $x, y$-coordinates of the vertices are the only degrees of freedom, the level set method requires determining functional values at every point of the two- or three-dimensional grid. Therefore, the level set method solves a higher-dimensional discrete problem, and in this sense is expected to have theoretically higher computational cost, especially on fine grids. Meanwhile, as the partial differential equation to be solved is a simple heat equation, the application of FFT allows its fast solution, 
Table F.4: CPU time (in seconds) of level set-based simulation of volume-preserving mean curvature flow

\begin{tabular}{|c|rrrrrr|}
\hline \multirow{2}{*}{ mesh resolution } & \multicolumn{6}{|c|}{ number of time steps } \\
\cline { 2 - 7 } & 15 & 30 & 60 & 120 & 240 & 480 \\
\hline $100 \times 100$ & 0.33 & 0.41 & 0.58 & 0.91 & 1.59 & 2.93 \\
$200 \times 200$ & 0.83 & 1.15 & 1.80 & 3.10 & 5.59 & 10.91 \\
$400 \times 400$ & 2.34 & 3.86 & 6.83 & 12.46 & 23.54 & 45.14 \\
$800 \times 800$ & 7.36 & 12.79 & 24.13 & 47.09 & 88.72 & 168.19 \\
$1600 \times 1600$ & 28.21 & 51.79 & 99.84 & 195.49 & 388.63 & 768.01 \\
\hline
\end{tabular}

as detailed in Methods section. Moreover, the intercalation example of Figure E.13A shows that the correct realization of gradient flow by the level set methods requires significantly smaller number of time steps to reach the equilibrium compared to the stiff evolution by vertex dynamics. Hence, the theoretically higher computational cost does not always imply longer computational time needed to reach the required solution.

Since it is not possible to cover all such case-dependent aspects, we use simple examples to report on the generic computational cost of both algorithms. All CPU times and memory usage tests reported in this section were conducted on a MacBook with $2 \mathrm{GHz}$ quad-core Intel Core i5 processor. Using the same setup as in Supplementary Section D.1, we measure runtime of our level set-based algorithm in terms of CPU time in seconds for various mesh-time configurations. Table F.4 and Figure F.15a show a moderate computational cost for finer mesh-time configurations over the vertex dynamics scheme, which is computationally cheaper in the sense that computations are only done at the vertices, contrary to all mesh nodes for the level set-based method. On the other hand, the cost increases linearly in both methods with respect to refinements: in time and each spatial direction for level set-based method, and in time and in number of vertices in vertex dynamics. The last statement is supported by Figure F.16, which shows the dependence of CPU time on time step size in vertex dynamics applied to test cases A and B from Supplementary Section E. Here CPU time was measured until a stopping criterion based on a stationary state is met; namely, distance by which each vertex in the network moved was measured in every time step and the code was terminated when all vertices moved by less than $10^{-12}$. The CPU times are longer for test case $\mathrm{A}$, where topology change has to be detected and implemented by a T1 swap. The cost difference from test case B, which does not involve any topology change, is not large but it is expected that it will become prominent when many $\mathrm{T} 1$ swaps are required during the evolution. In order to check whether level set-based simulation of configurations with topological changes affect computational cost, we employ test cases A and B from Supplementary Section E and measure runtime of our algorithm in terms of CPU time in seconds for various mesh-time configurations. Tables F.5 and F.6 as well as Figure F.15p indicate that there is no significant difference in computational cost between simulations with and without topological changes.

In addition, we investigated the dependence of computational cost on the number of simulated cells. The 
Table F.5: CPU time (in seconds) of level set-based algorithm for test case A

\begin{tabular}{|c|rrrrr|}
\hline \multirow{2}{*}{ mesh resolution } & \multicolumn{5}{|c|}{ number of time steps } \\
\cline { 2 - 6 } & 30 & 60 & 120 & 240 & 480 \\
\hline $200 \times 200$ & 38.60 & 59.26 & 106.51 & 211.13 & 416.63 \\
$400 \times 400$ & 154.99 & 321.23 & 631.96 & 997.70 & 1751.74 \\
$800 \times 800$ & 656.70 & 1262.54 & 2555.67 & 5491.67 & 8978.75 \\
\hline
\end{tabular}

Table F.6: CPU time (in seconds) of level set-based algorithm for test case B

\begin{tabular}{|c|rrrrr|}
\hline \multirow{2}{*}{ mesh resolution } & \multicolumn{5}{|c|}{ number of time steps } \\
\cline { 2 - 6 } & 30 & 60 & 120 & 240 & 480 \\
\hline $200 \times 200$ & 38.42 & 74.73 & 134.55 & 214.20 & 414.06 \\
$400 \times 400$ & 172.37 & 364.85 & 590.31 & 1018.51 & 1733.04 \\
$800 \times 800$ & 726.08 & 1351.05 & 2685.12 & 5641.49 & 12183.31 \\
\hline
\end{tabular}

domain $\Omega=[0,1] \times[0,1]$ was discretized using $800 \times 800$ grid points, time step was set to $\delta t=0.001$ and initial configuration was formed by "layers of bricks" with periodic boundary condition as shown in Figure F.15k. This initial network was constructed for 4, 16, 64 and 256 cells and evolved under uniform and constant interfacial tension and periodic boundary conditions for 50 time steps, when it approximately reached the stable honeycomb pattern shown in Figure F.15. Figure F.15 and Table F.7 show the required CPU times and memory for each of the four simulations. A quadratic function fitted tightly to the CPU time curve showing that the CPU times are proportional to the square of the number of cells. This matches with the theoretical prediction of quadratic complexity of auction dynamics in Jacobs et al. (2018), and implies significantly higher demands on computational resources than the linear dependence of vertex dynamics. Analogous behavior was obtained for different choices of discretization parameters.

Table F.7: Dependence of computational cost of level set-based algorithm on nu
\begin{tabular}{|c|r|r|}
\hline number of cells & CPU time (s) & memory (kB) \\
\hline 4 & 5.204 & 25,784 \\
16 & 22.007 & 85,812 \\
64 & 177.926 & 326,384 \\
256 & 2300.612 & 970,388 \\
\hline
\end{tabular}

Lastly, we measure memory usage in kilobytes of our algorithm for various mesh-time configurations using test cases A and B from Supplementary Section E. Table F.8 shows that there is no difference in memory usage between test cases $\mathrm{A}$ and $\mathrm{B}$. On the other hand, vertex dynamics solely requires storing of small arrays of the size proportional to the number of vertices. In our test cases A, B we have 64 cells with 128 vertices, entailing memory usage as small as approximately $45 \mathrm{kB}$, independently of choice of parameters. 

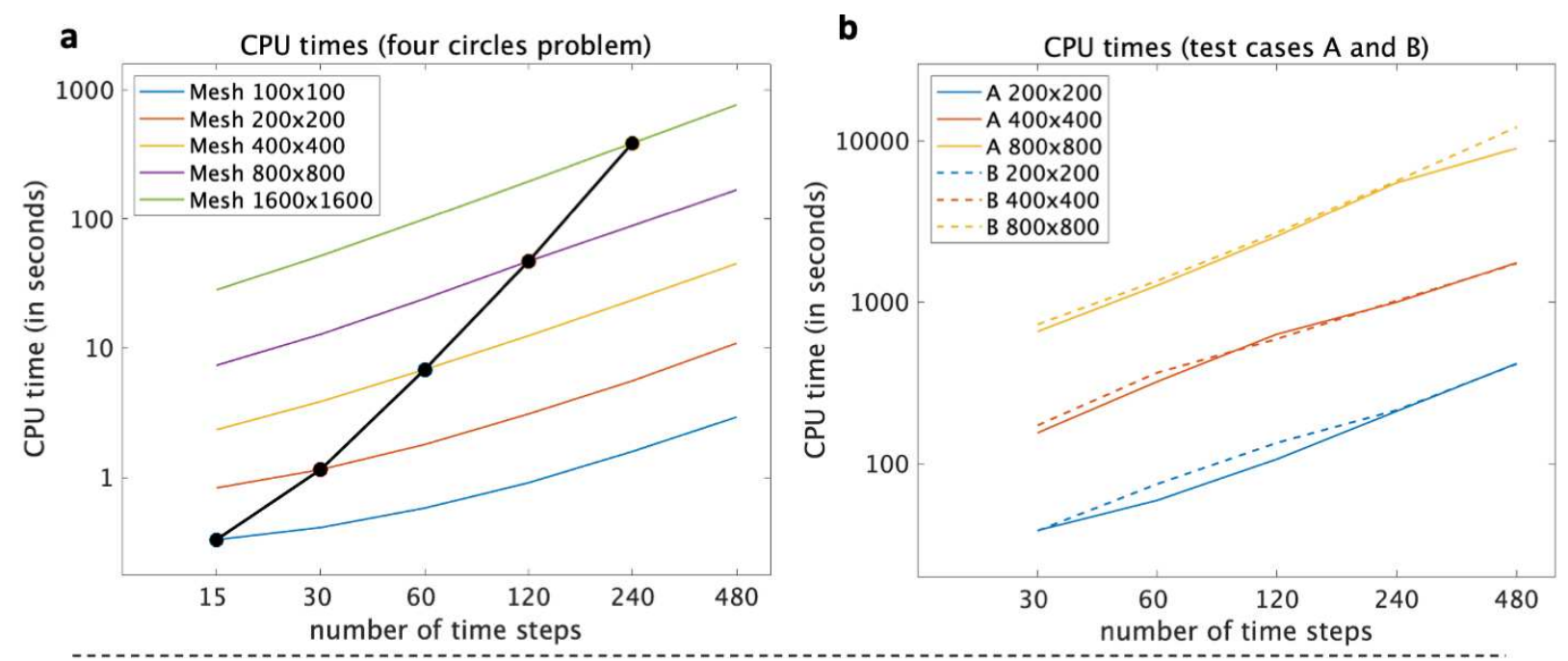

c

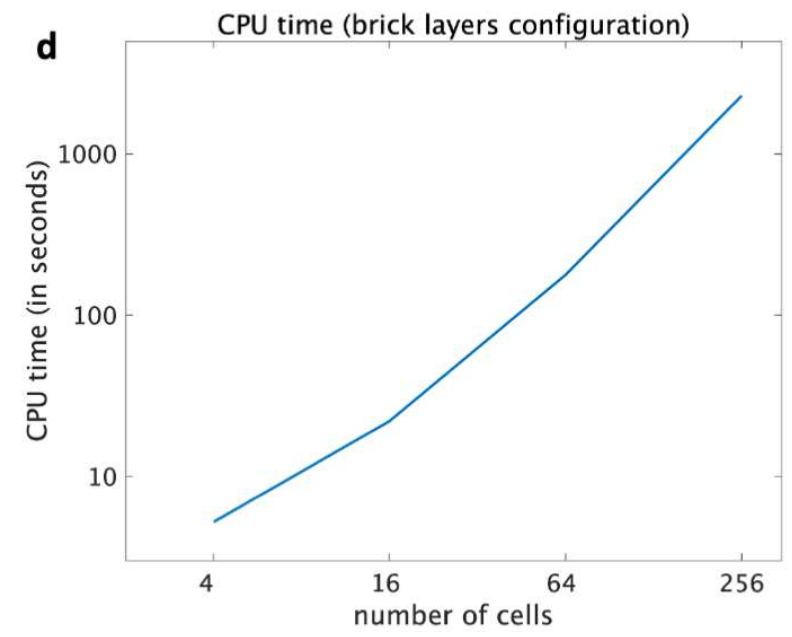

Figure F.15: Computational cost of level set-based algorithm. a,b, Log-log plot of CPU time (in seconds) of level set-based algorithm applied to volume-preserving mean curvature flow of Supplementary Section D.1 (a) and test cases A and B from Supplementary Section E (b) for various mesh-time configurations. Black points show the tendency of CPU times when number of discrete points is doubled both in time and the two spatial directions. The slope of black lines is approximately 8 , suggesting linear dependence. c, Initial configuration of 16 brick-like cells with periodic boundary condition and its evolution after 50 time steps using $800 \times 800$ mesh and time step $\delta t=0.001$. d Log-log plot of the dependence of CPU time on the number of cells obtained from the setup in $\mathbf{c}$.

Our level set-based algorithm has a significantly higher memory usage - the price for expressing complex cell geometries.

\section{Supplementary Section G. Cellular Mosaic Patterns}

Among the phenomena that occur in a heterotypic aggregate of embryonic cells are cell sorting, mixing, and formation of checkerboard patterns. In epithelial tissues, for example, when cells expressing different types 

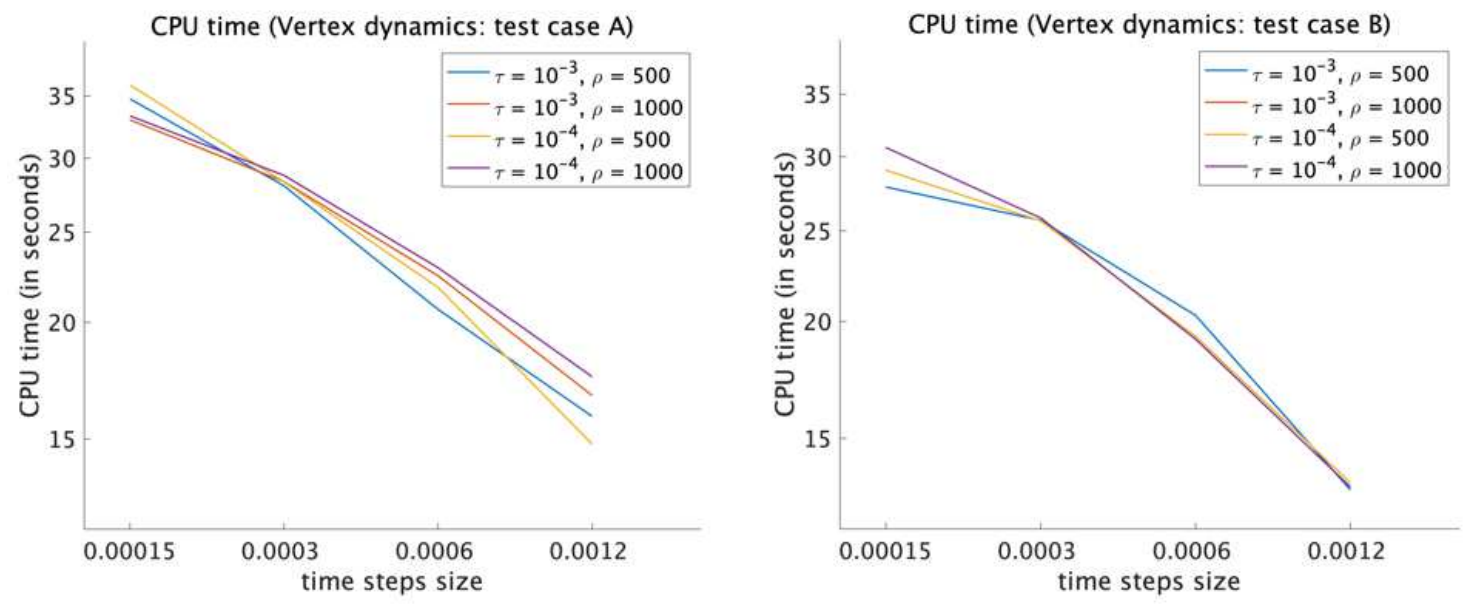

Figure F.16: Computational cost of vertex dynamics algorithm. Log-log plots of CPU times for executing the vertex dynamics algorithm on test case A (with topology change) and B (without topology change), showing increased cost when T1-swap is present.

Table F.8: Memory usage (in kilobytes) of level set-based algorithm for various mesh configurations

\begin{tabular}{|c|r|r|}
\hline mesh resolution & test case A & test case B \\
\hline $200 \times 200$ & 84,954 & 84,311 \\
$400 \times 400$ & 326,984 & 327,640 \\
$800 \times 800$ & $1,300,704$ & $1,299,728$ \\
\hline
\end{tabular}

of cadherin - a homophilic cell adhesion molecule - are mixed, these cells form separate aggregates (Nose et al. 1988). Similar segregation of cells occurs if cells expressing different amounts of the same cadherin are mixed (Steinberg and Takeichi, 1994). From these observations, cadherin quantity and affinity are thought to control tissue segregation and assembly. In contrast to cadherins, nectins prefer heterotypic binding to homotypic one, and their heterophilic interactions produce stronger cell-cell adhesions than their homophilic interactions. Owing to these properties of nectins, cells in mixed cultures expressing different nectins became arranged in a mosaic pattern (Togashi et al., 2011) (see Figure G.17).

Cell sorting begins with the formation of smooth chains of cells, followed by shortening of these chains into round masses, and finally annealing of the resulting masses (Brodland and Chen, 2000). This has been studied by Brodland (2002) from the viewpoint of the differential interfacial tension hypothesis, where selfrearrangement of embryonic cells and tissues are driven by differences in interfacial tensions. Finite elementbased simulations in Brodland and Chen (2000); Brodland (2004) showed that for an aggregate of two cell types, say, blue and red cells, sorting occurs only when interfacial tensions satisfy $\sigma_{\mathrm{BR}}>\frac{1}{2}\left(\sigma_{\mathrm{BB}}+\sigma_{\mathrm{RR}}\right)$; meanwhile a sufficient condition for mixing is $\sigma_{\mathrm{BR}}<\frac{1}{2} \sigma_{\mathrm{BB}}$ or $\sigma_{\mathrm{BR}}<\frac{1}{2} \sigma_{\mathrm{RR}}$.

We recreate these simulations using our level set-based approach. Consider an initial aggregate of 50 similar- 

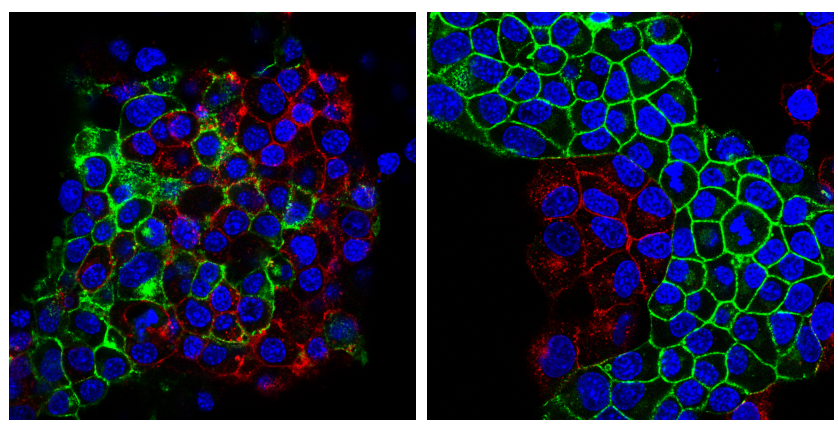

Figure G.17: Two different cell sorting mechanisms. (Left) Mosaic pattern of a mixed culture of cells expressing different nectins (green: nectin-1 (AF297665.1); red: nectin-3 (NM_021495.4); blue: cell nucleus). (Right) Segregated pattern of a mixed culture of cells expressing different cadherins (green: N-cadherin (NM_007664.5); red: E-cadherin (NM_009864.3); blue: cell nucleus).

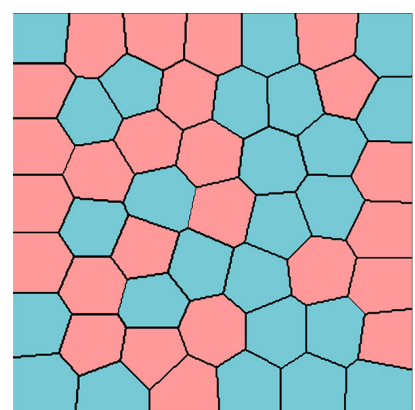

Initial Configuration

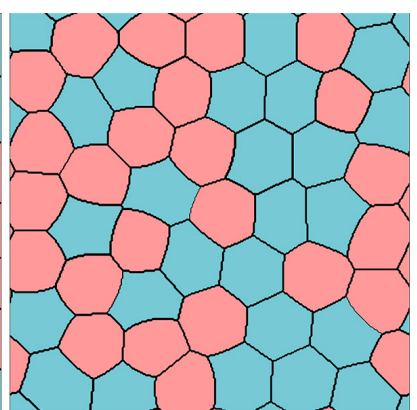

Partial Mixing

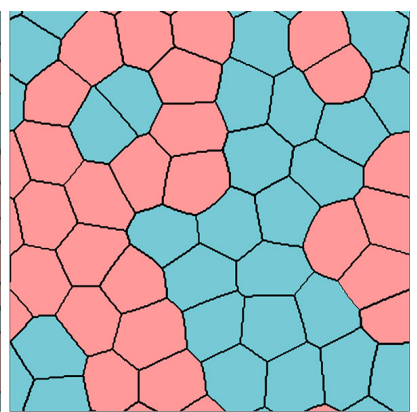

Partial Sorting

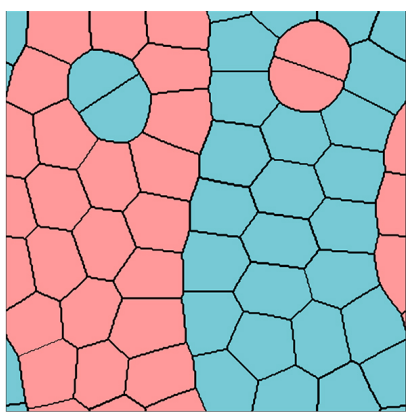

Strong Sorting

Figure G.18: Cell sorting via level set-based model. Initial aggregate of 25 blue and 25 red cells with interfacial tensions $\sigma_{\mathrm{BB}}=0.6$ and $\sigma_{\mathrm{RR}}=1.0$; its evolution generated by the level set-based approach for partial mixing when $\sigma_{\mathrm{BR}}=0.7$; partial sorting when $\sigma_{\mathrm{BR}}=1.1$; and strong sorting when $\sigma_{\mathrm{BR}}=2.0$.

sized cells where cell types are randomly assigned to 25 blue and 25 red cells (see Figure G.18 and Movie S11). We discretize the domain $\Omega=[0,1] \times[0,1]$ uniformly into $M=300 \times 300$ points, consider periodic boundary conditions, and set time step $\delta t=0.0008$. In this setup, we take interfacial tensions $\sigma_{\mathrm{BB}}=0.6$ and $\sigma_{\mathrm{RR}}=1.0$ over 300 time steps. We observe that when $\sigma_{\mathrm{BR}}=0.7$, partial mixing of blue and red cells occurs. Moreover, increasing this interfacial tension to $\sigma_{\mathrm{BR}}=1.1$ leads to partial sorting of different cell types; and further increasing to $\sigma_{\mathrm{BR}}=2.0$ results in strong sorting. Here we scale by $5 \%$ the interfacial tensions considered in Brodland (2002). Hence, our method is able to produce similar results as those of the finite element-based simulations in Figure 5 of (Brodland, 2002). The important difference is that our level set-based method naturally handles topological changes; while the FEM-based approach requires an ad hoc scheme, in particular, a boundary "flip" algorithm to handle cellular intercalations, which may cause problems for certain cellular configurations, leading to unnaturally distorted shapes of cells, in the same way as in the vertex dynamics model. 
On the other hand, employing the viewpoint of differential adhesion hypothesis, Katsunuma et al. (2016) hypothesized that relative intensities of $\beta$-catenin accumulations in mixed cultures of cells expressing nectin2 and N-cadherin (blue cells in Figure G.19) with various transfectants (red cells in Figure G.19) lead to different mosaic cellular patterning. In particular, a segregated pattern is formed when mixed with cells expressing nectin-2, N-cadherin, and E-cadherin where the adhesion strengths satisfy $\alpha_{\mathrm{RR}}>\alpha_{\mathrm{BR}}>\alpha_{\mathrm{BB}}$; a checkerboard pattern when mixed with cells expressing nectin-3 and N-cadherin resulting in $\alpha_{\mathrm{BR}}>\alpha_{\mathrm{RR}}=$ $\alpha_{\mathrm{BB}}$; and a football (kagome) pattern when mixed with cells expressing nectin-3, N-cadherin, and E-cadherin where $\alpha_{\mathrm{BR}}=\alpha_{\mathrm{RR}}>\alpha_{\mathrm{BB}}$. To confirm these hypothetical profiles of synergistic actions of nectins and cadherins on cellular patterning, we use our level set-based scheme and simulate the corresponding cell motions. In our simulations involving adhesion as the physical parameter, we always set the coefficients $\sigma$ in (1) as the reciprocal of the cell-cell adhesion strength $\alpha$, i.e., $\sigma=\alpha^{-1}$.

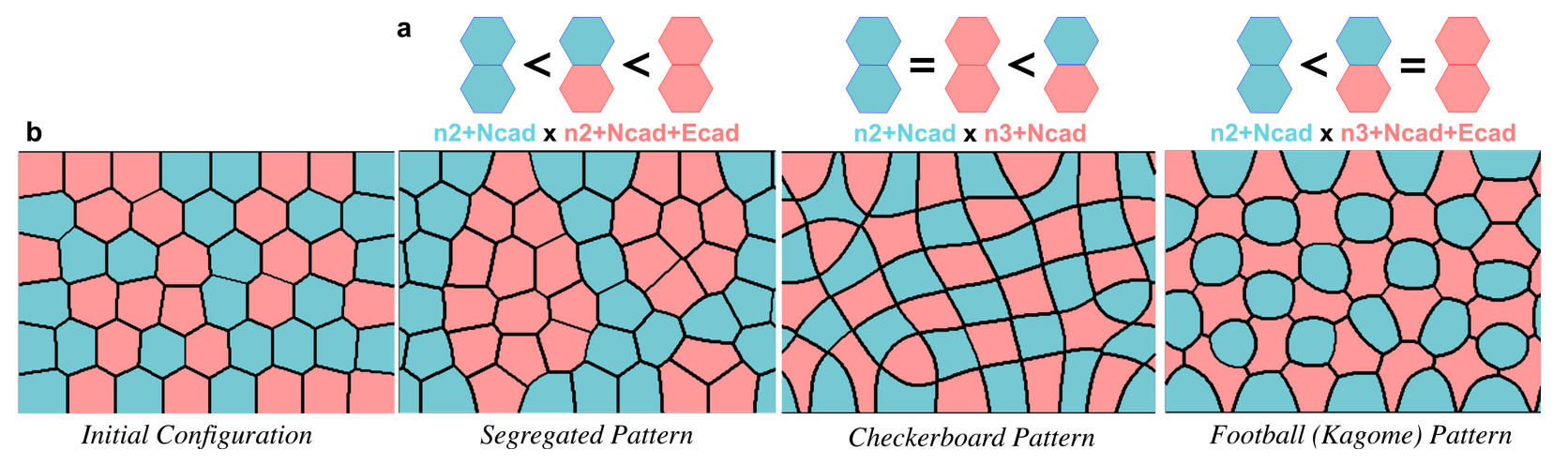

Figure G.19: Cellular patterns via level set-based model according to hypothetical profiles of synergistic actions of nectins and cadherins. a, Schematic diagrams of the relative intensities of the $\beta$-catenin accumulations (corresponding to relative adhesion strengths) in mixed cultures of various transfectants, resulting in different mosaic patterns. b, Initial 48-cell aggregate consisting of 24 blue cells expressing nectin-2 and N-cadherin; and its evolution via level set-based approach resulting in different mosaic patterns: a segregated pattern with red cells expressing nectin-2, N-cadherin, and E-cadherin; a checkerboard pattern with red cells expressing nectin-3 and N-cadherin; and football (kagome) pattern with red cells expressing nectin-3, N-cadherin, and E-cadherin.

We consider an initial 48-cell aggregate where cell types are randomly assigned to 24 blue and 24 red cells (see Figure G.19 and Movie S12). We take a computational domain $\Omega=[0,1] \times[0,1]$ discretized uniformly into $M=300 \times 300$ points and set time step $\delta t=0.001$. On the left and right boundaries, we impose periodic boundary conditions; while on the top and bottom boundaries, we prescribe a fixed adhesion strength of 0.10. Moreover, we consider time-dependent cell-cell adhesion strengths, which change piecewise linearly in time starting from $\alpha_{\mathrm{BB}}=\alpha_{\mathrm{BR}}=\alpha_{\mathrm{RR}}=1.0$ over 1500 time steps. More precisely, we increase the adhesion strengths starting from 1.0 in increments of size 0.25 keeping them constant for 150 time steps each time before the next increase, until they reach the value 3.0, which is kept constant for 450 time steps in order to completely equilibrate. Observe that when adhesion strengths $\alpha_{\mathrm{BB}}=\alpha_{\mathrm{BR}}=1.0$ are kept constant, while 
$\alpha_{\mathrm{RR}}$ is linearly changed to 3.0 , the blue and red cells segregate creating a final configuration similar to that produced by cell sorting. In particular, since $\alpha_{\mathrm{RR}}$ is large, the red cells strongly adhere to each other and sort out the blue cells. Moreover, for target adhesion strengths $\alpha_{\mathrm{BB}}=\alpha_{\mathrm{RR}}=1.0$ and $\alpha_{\mathrm{BR}}=3.0$, the stationary solution forms a checkerboard pattern. Note that a perfect checkerboard pattern is not attained due to the topological limitations near the boundary but red and green cells are still distributed in a fully alternating pattern. Finally, changing adhesion strengths to $\alpha_{\mathrm{BB}}=1.0$ and $\alpha_{\mathrm{BR}}=\alpha_{\mathrm{RR}}=3.0$ results in a football pattern. However, since there are equal number of cells for each type, only four blue cells surround each red cell and vice versa. If the initial aggregate has more red cells, enough for six of them to surround one blue cell, the final pattern will be much closer to the standard football pattern.

\section{Supplementary Section H. Cell Engulfment and 3D Simulations}

Here, we look into embryo morphogenesis where mammalian embryo self-organizes into a blastocyst, consisting of epithelial layer encapsulating the inner-cell mass (Maitre et al., 2016). Since asymmetrically divided 8-cell-stage blastomeres encompass both the morphogenesis and fate specification of the whole embryo, it is enough to consider a cell doublet as an initial configuration, which results in the blue cell (B) enveloping its neighboring red cell (R) in an entosis-like process within a white medium (W). Maître et al. (2016) showed that cells internalize only when differences in surface contractility exceed a predictable threshold, in particular, when $\sigma_{\mathrm{RW}} \geq \sigma_{\mathrm{BW}}+\sigma_{\mathrm{BR}}$, see equation (S16) in the Supplement of their paper.

We consider an initial configuration of a cell doublet on a cubical domain $\Omega=[0,1] \times[0,1] \times[0,1]$ discretized uniformly into $M=300 \times 300 \times 300$ points. We present a three-dimensional numerical simulation using our level set-based scheme with time step $\delta t=0.005$ and interfacial tensions satisfying the cell internalization condition. In particular, we start with $\sigma_{\mathrm{BR}}=1.6$, and $\sigma_{\mathrm{BW}}=\sigma_{\mathrm{RW}}=1.0$, then decrease the compaction parameter $\alpha$ from 0.8 to 0.25 through the reduction of $\sigma_{\mathrm{BR}}$ to 0.5 , followed by an increase in tension asymmetry $\delta$ from 1.0 to 1.6 through the change of $\sigma_{\mathrm{RW}}$ to 1.6 . The value of $\sigma_{\mathrm{B} W}=1.0$ is kept constant throughout the simulation. Results presented in Figure H.20 and Movie S13 confirm that our method produces similar results as in Maitre et al. (2016), and more importantly, it can handle simulations in higher dimensions without technical or theoretical complications. Indeed, the algorithm remains the same, it is

only solved on a 3-dimensional spatial mesh. This naturally entails a significant increase in the demand on computational resources and thus simulation of larger number of cells in 3D will require parallelization and other improvements in the basic code. 

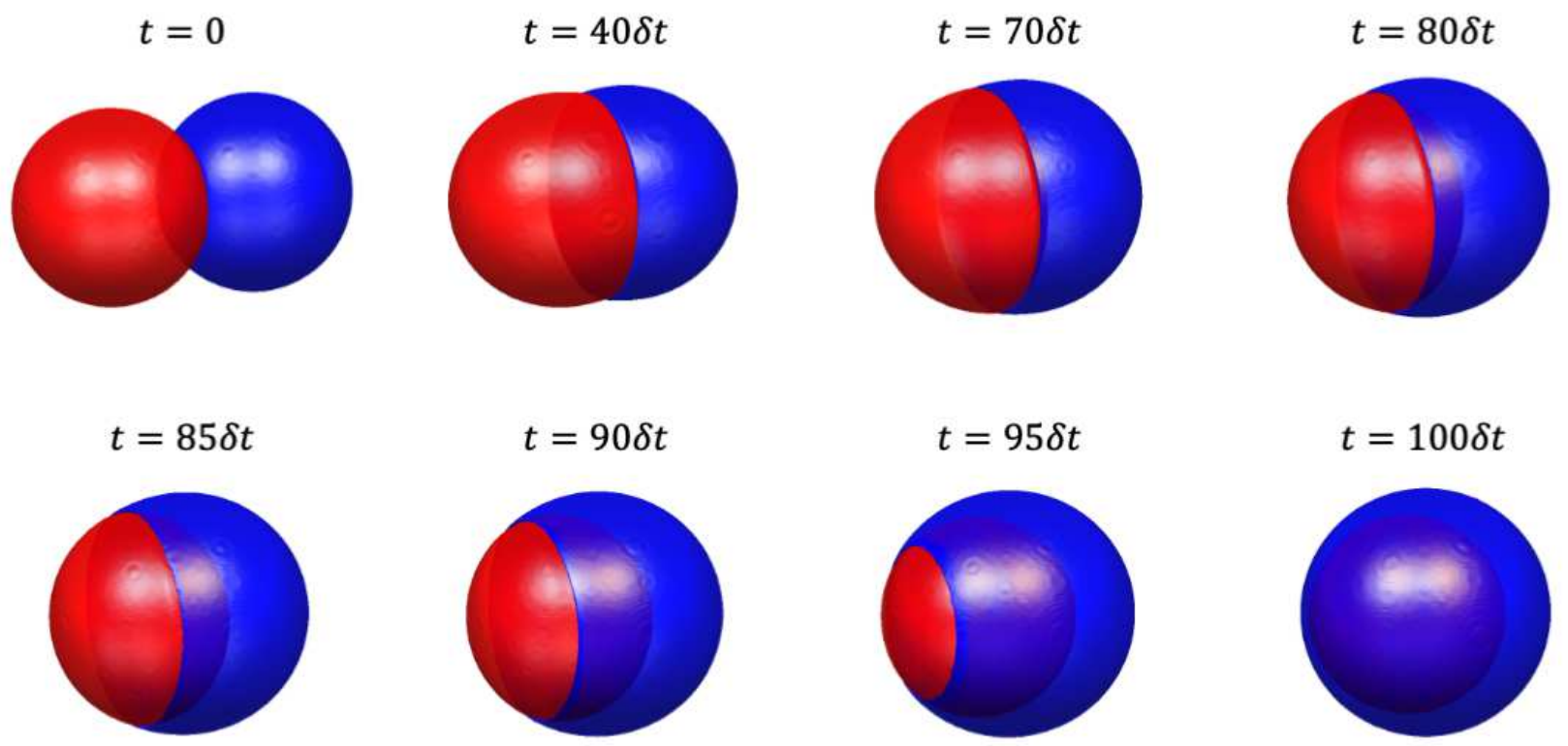

Figure H.20: Cell internalization via level set-based model. An initial cell doublet configuration $(t=0)$ and snapshots of its evolution using the level set-based approach with time step $\delta t=0.005$ and interfacial tensions $\sigma_{\mathrm{BR}}, \sigma_{\mathrm{BW}}, \sigma_{\mathrm{RW}}$ following the setup of Maître et al. (2016).

\section{Supplementary Section I. Further Simulations of Auditory Epithelium - Formation of Stable Pattern through Fluctuations}

Here we provide more details on the simulation of developing auditory epithelium regarding its dependence on the choice of initial condition. It was found that perfect checkerboard or mosaic pattern was not reached for every initial configuration of HCs and SCs prescribed at E14 stage, even when simulation parameters described in Section 2.4 were used. Figure I.21 and Movie S14 show numerical results obtained from a biased initial configuration where HCs and SCs cluster, contrary to the initial configuration in Figure 5 . The interfacial tensions are identical to those in the simulation shown in Figure 55, i.e., $\sigma_{\mathrm{SS}}=\sigma_{\mathrm{SH}}=\sigma_{\mathrm{HH}}=$ $\sigma_{\mathrm{SP}}=\sigma_{\mathrm{HP}}=1.0$ at E14 stage, only $\sigma_{\mathrm{SH}}$ changes to 0.6 at E16 stage, and finally $\sigma_{\mathrm{SS}}=0.3, \sigma_{\mathrm{SH}}=0.6$, $\sigma_{\mathrm{HH}}=1.0, \sigma_{\mathrm{SP}}=0.65$ and $\sigma_{\mathrm{HP}}=1.0$ at E18 stage. Under this setup, cells did not reach perfect checkerboard pattern at E16 and the final pattern at E18 was not the expected regular mosaic either.

In living tissues, various factors such as cellular fluctuations or mechanical forces (Cohen et al. 2020), induce relocation of cells. Figure $I .21$ s shows a simulation where a weak fluctuation within the range of $10 \%$ of the form

$$
\widetilde{\sigma}_{\mathrm{AB}}^{i j}(t)=\left(1+0.1 \min \left\{1, e^{-16(t-1)}\right\} \sin \frac{\bmod (7 i+9 j, 16 N)}{N} t\right) \sigma_{\mathrm{AB}} .
$$

was imposed on the interfacial tensions. Here, $\widetilde{\sigma}_{\mathrm{AB}}^{i j}$ is the modified interfacial tension between two cells with numbers $i$ and $j$, symbols $\mathrm{A}$ and $\mathrm{B}$ are to be replaced by any of the symbols $\mathrm{H}, \mathrm{S}$ or $\mathrm{P}$, and $N=25$ is 
the number of cells. In this case, perfect checkerboard pattern at E16 and the expected mosaic pattern at E18 were obtained. This simulation indicates that, independently of the cell configuration before stage E14, the combination of the affinity between HCs and SCs at stage E16 and of the fluctuations lead to a regular checkerboard pattern and subsequently to the desired highly organized pattern at E18. Elucidation of the mechanism of pattern formation requires further investigation and our level set-based approach can be a reliable tool for pursuing this research. Figure I.21; and Movie S14 depict results for the Nectin-3 $\mathrm{KO}$ simulation, i.e., $\sigma_{\mathrm{SH}}=0.9$ at E16. In this case, checkerboard pattern is not achieved at E16, even if fluctuations are imparted to interfacial tensions, and one confirms that the resulting final pattern coincides with the experimentally observed one (Figure 5:).
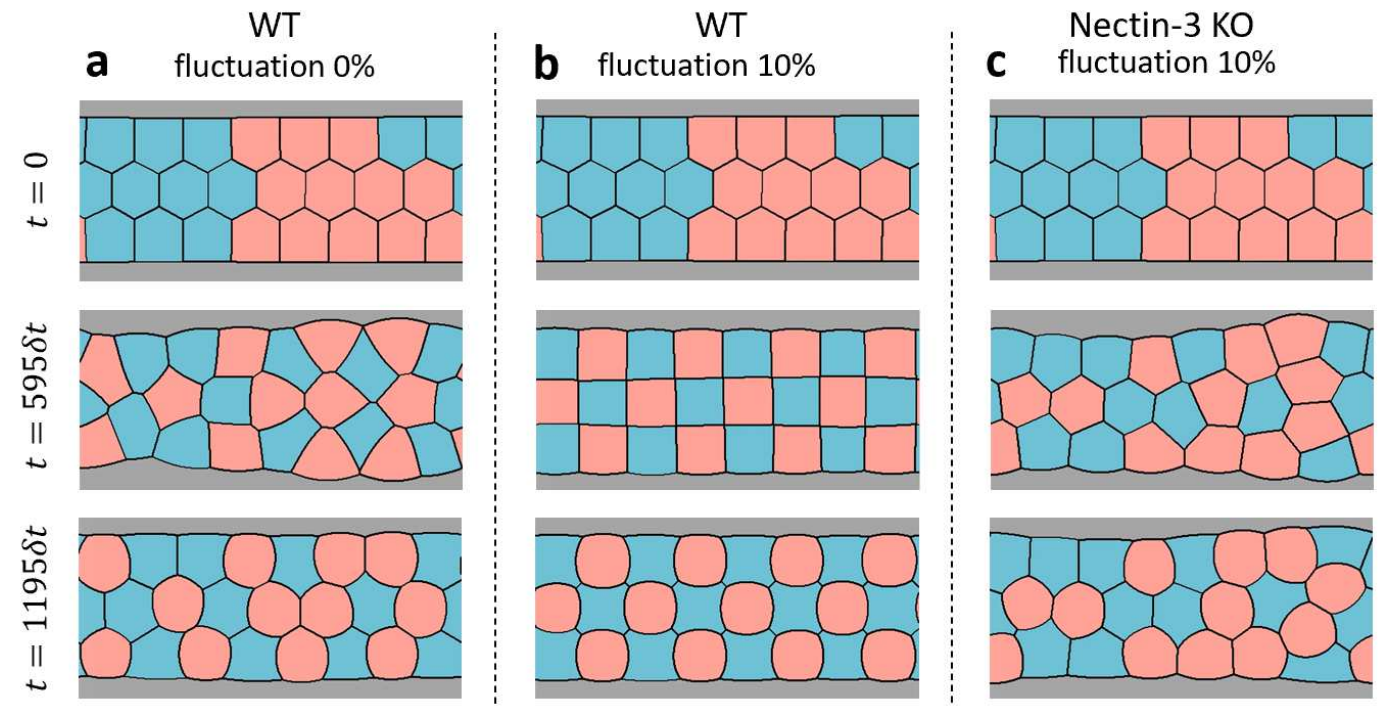

Figure I.21: Simulation of developing auditory epithelium starting from a biased initial state and impact of fluctuations. a, Simulation under the WT setup of Section 2.4 starting from an extremely biased initial configuration did not reach the expected mosaic pattern. b, On the other hand, weak fluctuations in surface tensions (within $10 \%$ of the original value) led to the stable attainment of regular patterns at E16 and E18 stages. c, The same fluctuations as in $\mathbf{b}$ did not improve the impaired final pattern obtained under the Nectin-3 KO setup. 


\section{Supplemental Movies.}
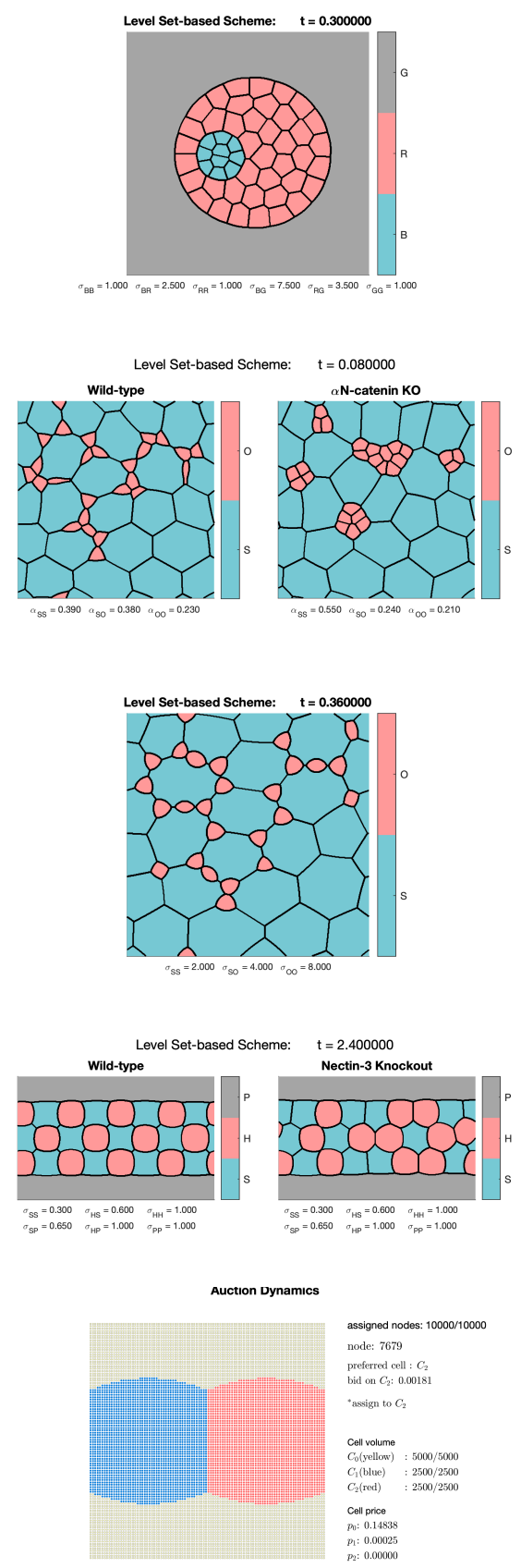

Movie S1: Total engulfment via level set-based model. An initial aggregate of 10 blue and 40 red cells surrounded by a medium and its evolution generated by our level set-based method, resulting in total engulfment of the blue cell mass by the red cell mass. Here the interfacial tensions are $\sigma_{\mathrm{BB}}=\sigma_{\mathrm{RR}}=1.0$, $\sigma_{\mathrm{BM}}=7.5, \sigma_{\mathrm{RM}}=3.5$, and $\sigma_{\mathrm{BR}}$ linearly changing from 7.5 at time $t=0$ to 2.5 at time $t=100 \delta t$ and then kept constant.

Movie S2: Simulations of cellular rearrangements of WT and $\alpha \mathrm{N}$-catenin KO olfactory epithelium from initial aggregate of 26 red OCs and 24 blue SCs to embryonic stages E14, E16, E18 until postnatal P1 stage obtained by the level set-based model using experimentally measured $\beta$-catenin intensities in Figure 5 of Katsunuma et al. (2016).

Movie S3: Simulation of a developing olfactory epithelium via level setbased model using hypothetical values of interfacial tensions at postnatal stage. Shapes of cells similar to those observed in experiments (Figure 3 P1) were reproduced.

Movie S4: Simulations of cellular rearrangements of WT and Nectin-3 KO embryonic auditory epithelium from initial aggregate of $12 \mathrm{HCs}$ (red), 12 SCs (blue), and one gray pillar cell, generated employing level set-based model.

Movie S5: An animation of auction dynamics to preserve cell volume in one time step of cellular rearrangement in a 3-cell aggregate. The video includes details of the bidding process, price and volume changes, together with nodal assignments. 

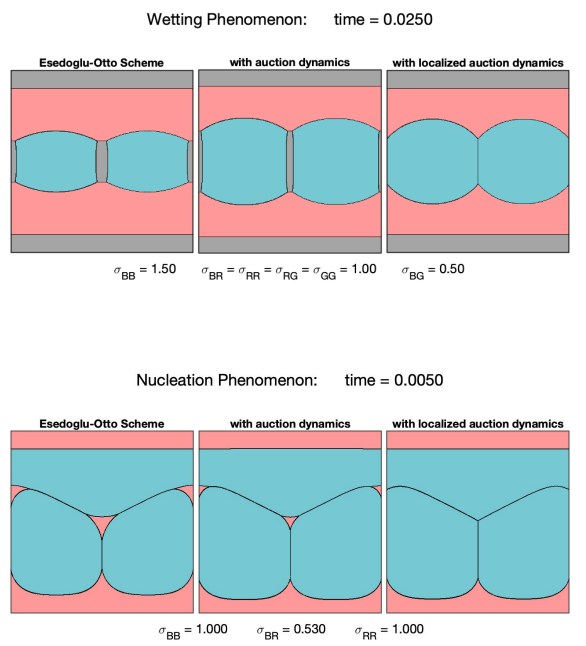

$\sigma_{\mathrm{BB}}=1.000 \quad \sigma_{\mathrm{BR}}=0.530 \quad \sigma_{\mathrm{PR}}=1.000$
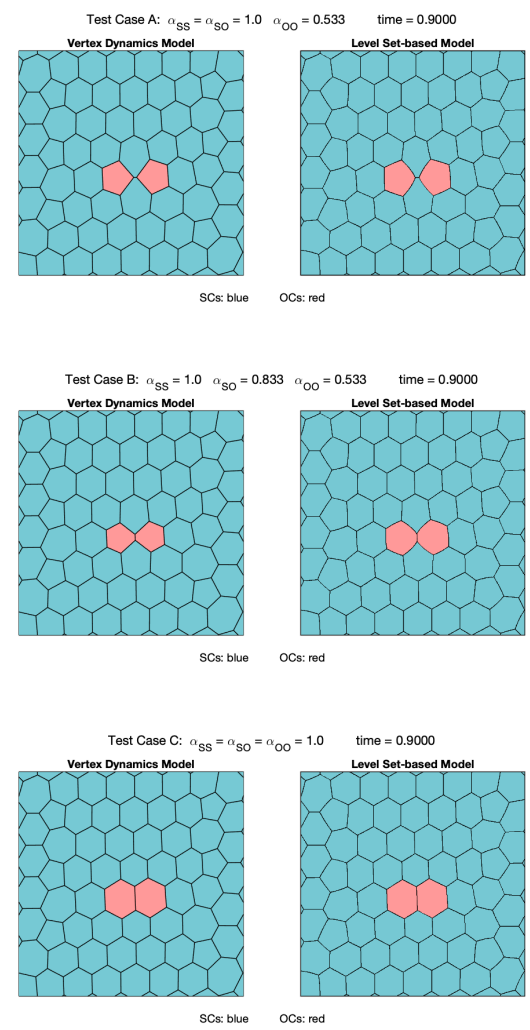

Cell Sorting via Level Set-based Model: $\quad$ time $=0.2400$

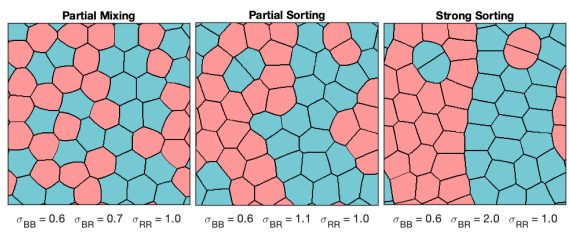

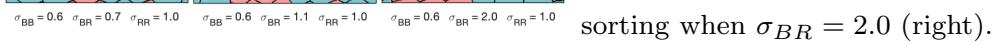

Movie S6: Wetting phenomenon in the numerical implementation of level setbased methods. Initial 4-cell configuration and its evolution under a wetting condition due to violation of $\sigma$-triangle inequality using Esedoglu-Otto scheme only (left); EO scheme with auction dynamics algorithm (center); and EO scheme with localized auction dynamics (right).

Movie S7: Nucleation phenomenon in the numerical implementation of level set-based methods. Initial 4-cell configuration and its evolution at $t=10 \delta t$ using Esedoğlu-Otto algorithm (left) resulting in nucleation of red cell at the blue triple junction; EO scheme with auction dynamics (center) where red cell splitting persists; and EO scheme with localized auction dynamics (right) which preserves cell connectivity.

Movie S8: Vertex dynamics vs. Level set-based model on Test Case A, where adhesion strengths are $\alpha_{S S}=\alpha_{S O}=1.0$ and $\alpha_{O O}=0.533$ leading to cellular intercalation. Initial aggregate of $62 \mathrm{SCs}$ (blue) and 2 OCs (red), and its evolution via vertex dynamics model with volume penalty $\rho=500$ (left) and level set-based approach (right).

Movie S9: Vertex dynamics vs. Level set-based model on Test Case B, with adhesion strengths $\alpha_{S S}=1.0, \alpha_{S O}=0.833$ and $\alpha_{O O}=0.533$, where no intercalation occurs. Initial aggregate of $62 \mathrm{SCs}$ (blue) and 2 OCs (red), and its evolution via vertex dynamics model with volume penalty $\rho=500$ (left) and level set-based approach (right).

Movie S10: Vertex dynamics vs. Level set-based model on Test Case C, where adhesion strengths are $\alpha_{S S}=\alpha_{S O}=\alpha_{O O}=1.0$, which shortens OO-junction. Initial aggregate of $62 \mathrm{SCs}$ (blue) and $2 \mathrm{OCs}$ (red), and its evolution via vertex dynamics model with volume penalty $\rho=500$ (left) and level set-based approach (right).

Movie S11: Cell sorting via level set-based model. Initial aggregate of 25 blue and 25 red cells with interfacial tensions $\sigma_{B B}=0.6$ and $\sigma_{R R}=1.0$; its evolution generated by the level set-based approach for partial mixing when $\sigma_{B R}=0.7$ (left); partial sorting when $\sigma_{B R}=1.1$ (center); and strong 
Movie S12: Cellular patterns via level set-based model according to

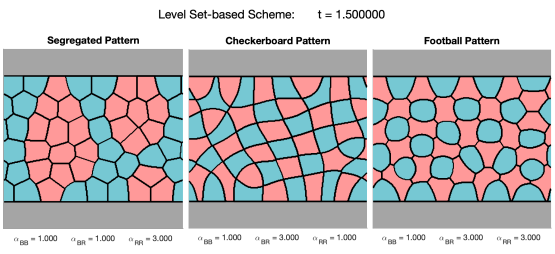

hypothetical profiles of synergistic actions of nectins and cadherins. Initial 48-cell aggregate consisting of 24 blue cells expressing nectin-2 and Ncadherin; and its evolution via level set-based approach resulting in different mosaic patterns: a segregated pattern (left) with red cells expressing nectin2, N-cadherin, and E-cadherin; a checkerboard pattern (center) with red cells expressing nectin-3 and N-cadherin; and football (right) pattern with red cells expressing nectin-3, N-cadherin, and E-cadherin.

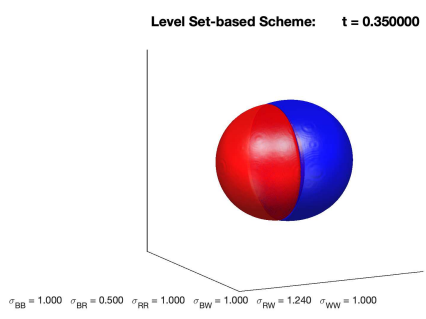

Movie S13: Cell internalization via level set-based model. An initial cell doublet configuration and its evolution using a level set-based approach with time step $\delta t=0.005$ and interfacial tensions $\sigma_{\mathrm{BR}}, \sigma_{\mathrm{BW}}, \sigma_{\mathrm{RW}}$ following the setup of Maître et al. (2016).

Movie S14: Simulation of developing auditory epithelium starting from a biased initial state, showing also the impact of fluctuations in interfacial tensions. Left panel shows WT model simulation starting from an extremely biased initial configuration, middle panel shows the same simulation with added tension fluctuations of size up to $10 \%$, and the right panel shows Nectin-3 KO model simulation with the same type of fluctuations. 


\section{Supplementary Files}

This is a list of supplementary files associated with this preprint. Click to download.

- MovieS01engulfment.mp4

- MovieS02olfactory.mp4

- MovieS03olfactoryE18.mp4

- MovieS04auditory.mp4

- MovieS05auction.mp4

- MovieS06wetting.mp4

- MovieS07nucleation.mp4

- MovieS08testcaseA.mp4

- Movies09testcaseB.mp4

- MovieS10testcaseC.mp4

- MovieS11sorting.mp4

- Movies12patterning.mp4

- Movies13internalization.mp4

- MovieS14AEfluctuation.mp4 